\title{
RBFOX2 IS CRITICAL FOR MAINTAINING ALTERNATIVE POLYADENYLATION PATTERNS AND MITOCHONDRIAL HEALTH IN RAT MYOBLASTS
}

Jun $\mathrm{Cao}^{1+}$, Sunil K. Verma ${ }^{1}$, Elizabeth Jaworski ${ }^{1}$, Stephanie Mohan ${ }^{1}$, Chloe K. Nagasawa ${ }^{1}$, Kempaiah Rayavara ${ }^{2}$, Amanda Sooter ${ }^{3}$, Sierra N. Miller ${ }^{4}$, Richard J. Holcomb $^{1}$, Ping $\mathrm{Ji}^{1}$, Nathan D. Elrod ${ }^{1}$, Eda Yildirim ${ }^{5}$, Eric J. Wagner ${ }^{1,6}$, Vsevolod Popov ${ }^{7}$, Nisha J. Garg ${ }^{2}$, Andrew L. Routh*,1,6 and Muge N. Kuyumcu-Martinez ${ }^{*}, 1,4$

1 Department of Biochemistry and Molecular Biology, University of Texas Medical Branch, Galveston, Texas

2 Department of Microbiology and Immunology, University of Texas Medical Branch, Galveston, Texas

3 School of Medicine, University of Texas Medical Branch, Galveston, Texas

4 Department of Neuroscience, Cell Biology and Anatomy, University of Texas Medical Branch, Galveston, Texas

5 Department of Cell Biology, Duke University School of Medicine

6 Sealy Centre for Structural Biology and Molecular Biophysics, University of Texas Medical Branch, Galveston, Texas

7 Department of Pathology, University of Texas Medical Branch, Galveston Texas 
+Present address: Cardiology Department, Boston Children's Hospital, Harvard

Medical School, Boston, MA 02115

${ }^{* *}$ Co-corresponding authors

Lead contact: Muge N. Kuyumcu-Martinez, nmmartin@utmb.edu, Department of Biochemistry and Molecular Biology; Institute for Translational Sciences; Department of Neuroscience, Cell Biology, and Anatomy, University of Texas Medical Branch, Galveston, Texas.

Andrew L. Routh, alrouth@utmb.edu, Department of Biochemistry and Molecular Biology; Sealy Centre for Structural Biology and Molecular Biophysics, University of Texas Medical Branch, Galveston, Texas. 


\section{SUMMARY}

RBFOX2, which has a well-established role in alternative splicing, is linked to heart diseases. However, it is unclear whether RBFOX2 has other roles in RNA processing that can influence gene expression/function in muscle cells, contributing to disease pathology. Here, we employed both 3'-end and nanopore cDNA sequencing to reveal a previously unrecognized role for RBFOX2 in maintaining alternative polyadenylation (APA) signatures in myoblasts. We found that RBFOX2-mediated APA modulates both mRNA levels and isoform expression of a collection of genes including contractile and mitochondrial genes. We identified the key muscle-specific contractile gene, Tropomyosin 1 and essential mitochondrial gene, S/c25a4 as APA targets of RBFOX2. Unexpectedly, depletion of RBFOX2 adversely affected mitochondrial health in myoblasts that is in part mediated by disrupted APA of mitochondrial gene S/c25a4. Mechanistically, we found that RBFOX2 regulation of S/c25a4 APA is mediated through consensus RBFOX2 binding motifs near the distal polyadenylation site enforcing the use of the proximal polyadenylation site. In sum, our results unveiled a new role for RBFOX2 in fine tuning expression levels of mitochondrial and contractile genes via APA in myoblasts relevant to heart diseases. 
Keywords: RBFOX2, alternative polyadenylation, mitochondria, S/c25a4, Tropomyosin 1 , nanopore sequencing, $\operatorname{poly}(A)$ sequencing

Non-standard Abbreviations and Acronyms:

AS

PAC-seq

APA

$\mathrm{KD}$

3'UTR

PAC

DPAC

PAS

dPAS

pPAS

ANT1 (S/c25a4)

MFN1 alternative splicing

poly(A)-ClickSeq

alternative polyadenylation

knock down

3'untranslated region

poly(A)-cluster

differential-poly(A)-clustering

Poly(A) site

distal poly(A) site

proximal poly $(A)$ site

ATP/ADP translocator (mitochondrial)

protein

Mitofusin 


\section{INTRODUCTION}

RBFOX2 belongs to a family of RNA binding proteins including RBFOX1 and RBFOX3 (neuron specific). RBFOX2 has a well-characterized role in alternative splicing (AS) regulation of pre-mRNAs that can affect gene expression and function. RBFOX2 controls AS by binding to a highly conserved motif (U)GCAUG in intronic and/or exonic regions of pre-mRNAs (Huang et al., 2012; Lovci et al., 2013; Sun et al., 2012; Yeo et al., 2009) and regulates AS in a large complex with other splicing regulators (Damianov et al., 2016). RBFOX2 has been linked to congenital heart defects (Homsy et al., 2015; Verma et al., 2016b), heart failure (Wei et al., 2015b), cardiac complications of diabetes (Nutter et al., 2017; Nutter et al., 2016b) and cardiac arrhythmias in Myotonic Dystrophy (Misra et al., 2020). RBFOX2 has been shown to be important for skeletal muscle development and function (Singh et al., 2018; Singh et al., 2014). Due to its important role in AS, the majority of studies have been on identification of RBFOX2-regulated AS events in heart and skeletal muscle (Runfola et al., 2015; Singh et al., 2018; Verma et al., 2016a). However, it remains unclear how loss of RBFOX2 contributes to heart and skeletal muscle defects. Recent studies have suggested that RBFOX2 binds within the 3'UTR of transcripts (Lovci et al., 2013; Wang et al., 2008; Weyn-Vanhentenryck et al., 2014) in proximity with poly(A) sites (PASs) (Wang et al., 2008). The consequences of this binding are unknown and a role for RBFOX2 in cleavage and polyadenylation of premRNAs has not yet been demonstrated.

Mammalian 3'end processing involves the cleavage of the pre-mRNA near the PASs followed by polyadenylation of the cleaved RNA. This process is complex and requires both cis-acting elements in the RNA and trans-acting proteins that bind to 
these elements and regulate cleavage and polyadenylation. Greater than $50 \%$ of genes in the human genome present more than one PASs and thus can generate multiple mRNA isoforms defined by different 3' termini (Tian et al., 2005). Alternative polyadenylation (APA) is defined by the differential usage of these PASs in a given mRNA (Shi, 2012). APA can regulate generation of multiple gene isoforms and impact mRNA expression and homoeostasis (Derti et al., 2012). There are two major types of APA: tandem-APA and splicing-APA. Tandem-APA is mediated by the preferential usage of tandem PASs within a 3'UTR. This type of APA can affect 3'UTR length modulating the presence of cis-acting regulatory elements and/or microRNA binding sites that can control mRNA stability, localization or translation (Di Giammartino et al., 2011). Splicing-APA can lead to generation of multiple protein isoforms with distinct functions and properties via AS of the terminal exons that have PASs (Cooke et al., 1999). Tight regulation of APA is essential for normal growth and development and as a result aberrant APA has been linked to many disease states including heart failure, cancer, and muscle disease (Batra et al., 2014; Creemers et al., 2016; Masamha et al., 2014).

In this study, we investigated whether RBFOX2 influences APA decisions in H9c2 myoblasts. We used long-read nanopore cDNA sequencing and Poly(A)ClickSeq (PAC-seq) (Routh et al., 2017), which allow precise genome wide determination of APA and can quantify gene expression and isoform generation. We identified 233 genes with APA changes upon RBFOX2 depletion in H9c2 myoblasts. $43 \%$ of these genes also exhibited mRNA level changes. Further analysis showed that 136 genes that displayed 3'UTR length changes in RBFOX2 depleted myoblasts are 
known targets of microRNAs, suggesting that modulation of 3'UTR length can impact protein production. We identified RBFOX2 as a regulator of both tandem and splicingAPA, impacting gene expression and isoform generation. Importantly, our results identified mitochondrial gene Slc25a4 and contractile gene Tropomyosin 1, as APA targets of RBFOX2. Investigating how RBFOX2 impacts APA-mediated rat Slc25a4 expression revealed that RBFOX2 binding motifs located $<79$ nt from the distal polyA site in Slc25a4 are inhibitory for polyA site usage, negatively modulating S/c25a4 protein levels. Moreover, the loss of Slc25a4 APA regulation in RBFOX2-depleted cells correlated with a striking increase in mitochondrial membrane potential and swelling. In summary, we report a new role for RBFOX2 in tuning APA patterns and mitochondrial health.

\section{RESULTS}

RBFOX2 depletion alters global alternative polyadenylation patterns of pre-mRNAs in $\mathrm{H} 9 \mathrm{c} 2$ myoblasts

RBFOX2 binds to the 3'UTR of transcripts (Lovci et al., 2013; Wang et al., 2008; Weyn-Vanhentenryck et al., 2014) near to the PASs (Wang et al., 2008), suggesting a potential role in APA. We have previously shown that H9c2 myoblasts successfully recapitulated RBFOX2-mediated AS regulation observed in mouse hearts (Nutter et al., 2016a), making it a good cell culture model to study RBFOX2 function in vitro. Thus, we tested whether RBFOX2 influences APA patterns in rat H9c2 myoblasts. We treated H9c2 cells with scrambled or Rbfox2-specific siRNAs and validated KD of RBFOX2 by Western blot (WB) (Fig. 1A). We then isolated RNA from these cells and performed PAC-seq 
(Routh et al., 2017) and analyzed the data using the differential-poly(A)-clustering (DPAC) pipeline (Routh, 2019a). We detected 101,338 unique PASs with at least 5 unique reads mapping from at least one sample. Multiple PASs occurring within 10nts were clustered together to yield 81,035 unique PASs. We further filtered out all PASs that did not map to 3'UTRs already annotated in the UCSC RefSeq database. After instituting these filters, remaining 22,768 PASs mapped to 10,964 unique mRNA transcripts, of which 5,317 contained multiple PASs and 556 contained multiple terminal exons (TE). DPAC reported 233 APA changes in RBFOX2 depleted myoblasts based on the criteria that differential PAS usage was reported if an individual PAS has a fold change of $>1.5$, and results with an Independent Hypothesis Weighted (Ignatiadis et al., 2016) multiple testing p-adjusted value of $<0.1$ (Fig. 1B, Supp. Data File 1).

We investigated what types of APA were affected upon RBFOX2 depletion in H9c2 cells. Out of 233 APA events, 213 were tandem-APA, 17 were splicing-APA, and 3 displayed both splicing and tandem-APA (Fig. 1B) (Supp. Data File 2). Using the Gene Ontology (GO) biological function analysis of these APA events, we found an enrichment of genes involved in striated muscle development and regulation of cell organization (Fig. 1C) (Supp. Data File 3). This is consistent with RBFOX2's involvement in congenital heart defects (Homsy et al., 2015; Verma et al., 2016b) and muscle development (Singh et al., 2018; Singh et al., 2014).

\section{RBFOX2-mediated splicing-APA is important for expression of rat Tropomyosin 1 muscle-specific isoforms}

Based on the GO analysis of RBFOX2 regulated APA events, we first investigated 
genes that have roles in striated muscle development that are impacted by RBFOX2 mediated splicing-APA (Supp. Data File 2 and Data File 3). Of the 17 splicing-APA changes identified in RBFOX2 depleted myoblasts, Tropomyosin 1 gene (Tpm1) has an essential role in striated muscle development and function and undergoes a dramatic splicing-APA change in RBFOX2 depleted cells (Supp. Data File 2 and Data File 3). Tpm1 is an essential gene required for myofibril organization (Thomas et al., 2010), muscle contraction (Wolska and Wieczorek, 2003), and cardiac development (England et al., 2017). Tpm1 has very complex AS and APA patterns and many isoforms that are tissue specific and developmentally regulated (Gooding and Smith, 2008). Thus, we further studied splicing-APA regulation of rat Tpm1 by RBFOX2.

PAC-seq identified 2 PASs located in 2 different last exons (exon 9b and exon 9d) that are utilized in $\mathrm{H} 9 \mathrm{c} 2$ myoblasts (Fig. 2A). Tpm1 gene isoforms ending with exon 9b are important for muscle contraction in muscle cells (Lin et al., 2008) (short or musclespecific transcripts); whereas gene isoforms ending with exon $9 \mathrm{~d}$ are ubiquitously expressed to support cytoskeleton function (long or non-muscle transcripts). The usage of PAS in muscle specific exon 9b diminished in RBFOX2 knocked down (KD) myoblasts (Fig. 2A).

To confirm the results obtained by PAC-seq, we used nanopore cDNA sequencing as an orthogonal approach to investigate full length Tpm1 isoforms and their expression in response to changes in RBFOX2 levels. We performed nanopore cDNA sequencing in control vs RBFOX2 KD cells and obtained high quality and full-length nanopore reads that were mapped to the rn6 genome using Minimap2 (Li, 2018) yielding an average of 550,000 mapped reads spanning 3000-4300 unique mRNAs (Supp. Fig. 1 and Supp. 
Table 1). Reads were mapped to specific rat Tpm1 exons (Fig. 2B, black rectangles), identifying 22 different full length Tpm1 transcripts in control H9c2 myoblasts (Supp. Fig. 2). These Tpm1 transcripts ended with different terminal exons (exon 9a, exon 9b or exon 9d) in control cells (representative images shown in Fig. 2B). Upon depletion of RBFOX2, we observed a strong reduction in the diversity of Tpm1 isoforms with only nine different full- length transcripts that ended almost exclusively with exon 9d (Fig. 2B and Supp. Fig. 3), consistent with reduced PAS usage in exon 9b in RBFOX2 KD cells (Fig. 2A). These indicate that RBFOX2 KD reduces expression of muscle-specific isoforms that end with exon $9 b$.

To further validate APA changes in muscle-specific (short) isoforms of Tpm1, we designed primers spanning Tpm1 exon7 and exon 9b or exon 7 and exon 9d (Supp. Table 2). The levels of Tpm1 muscle-specific transcripts were dramatically decreased in RBFOX2 depleted cells (Fig. 2C). Consistent with reduced levels of muscle-specific Tpm1 isoforms, TPM1 protein levels were reduced by $45 \%$ in RBFOX2 KD cells (Fig. 2D). Importantly, mRNA levels of Tpm1 non-muscle (long) transcripts did not change upon RBFOX2 KD (Fig. 2E).

We performed rescue experiments by ectopically expressing GFP-tagged RBFOX2 in RBFOX2 KD H9c2 cells. Rbfox2 siRNA treatment caused effective KD of RBFOX2 in H9c2 cells, and GFP-RBFOX2 protein was expressed at lower levels than the endogenous RBFOX2 due to targeting by RBFOX2 specific siRNAs (Fig. 2F, lane 3 vs 1). Even low levels of GFP-RBFOX2 partially rescued the expression of musclespecific Tpm1 transcripts (Fig. 2G). GFP-RBFOX2 expression did not affect expression of non-muscle Tpm1 isoforms (Fig. 2H). Importantly, Tpm1 regulation by RBFOX2 was 
specific because we did not observe dramatic changes in APA patterns of rat Tpm2, Tpm3 and Tpm4 in RBFOX2 KD myoblasts (Supp. Fig. 4).

These data show that RBFOX2 controls expression of muscle-specific isoforms of essential contractile gene Tpm1 via splicing-APA. Moreover, our results also demonstrate that nanopore sequencing is an effective method in revealing complex and coordinated APA and AS patterns and validating AS and APA variants.

\section{RBFOX2-mediated tandem-APA alters 3'UTR length and mRNA levels}

Having established splicing-APA mediated regulation of Tpm1 pre-mRNA by RBFOX2, we sought to identify novel tandem-APA events in genes that may be critical for muscle development and function. We found that the majority of the APA changes in RBFOX2 depleted cells were tandem-APA (Fig. 1B), which can modulate the 3'UTR length and, in turn, gene expression levels. Analysis of PAC-seq data revealed that 129 out of $216(59 \%)$ APA changes led to 3'UTR shortening (Supp. Data Files 1 and 4) and 64 out of $216(29.6 \%$ ) led to 3'UTR lengthening (Supp. Data Files 1 and 5) in RBFOX2 depleted cells (Fig. 3A). There was a smaller subset that included 23 genes that contained 3 or more PASs giving rise to complex changes resulting in both lengthening and shortening of $3^{\prime}$ UTRs in RBFOX2 KD cells due to the modulation of levels of both the most distal and most proximal PASs relative to an intermediate PAS (Fig. 3A) (Supp. Data Files 1 and 6). It has been well-established that 3'UTRs harbor binding sites for microRNAs that can influence mRNA translation and/or stability. We used TargetScan to determine whether genes with 3'UTRs length changes harbor known targets of microRNAs. We found that out of 216 tandem-APA events that display 3'UTR length 
changes in RBFOX2 KD myoblasts, the majority (136 genes) displayed microRNA binding sites in their 3'UTRs (Fig. 3B) (Supp. Data File 7). These results suggest that changes in 3'UTR length mediated via RBFOX2 depletion could affect microRNA-mediated regulation of these genes.

Given that 3'UTR length can alter mRNA stability, we wondered if genes that exhibit APA changes upon RBFOX2 KD also display expression level changes. There were 491 mRNA level changes (fold Change $>1.5$, p-adjust $<0.1$ ) in RBFOX2 KD myoblasts (Fig. 3C) (Supp. Data File 8). GO analysis (Kuleshov et al., 2016) of genes that are downregulated in RBFOX2 KD myoblasts revealed mitochondria, mitochondrial membrane and mitochondrial respiratory chains as affected processes (Fig. 3D) (Supp. Data File 9). There were 26 mitochondrial genes that were downregulated in RBFOX2 depleted myoblasts (Supp. Data File 9), Importantly, our analysis of differential gene expression also revealed that 99 of the $216(46 \%)$ genes with tandem-APA changes in RBFOX2 depleted myoblasts also exhibited changes in mRNA levels (Fig. 3E). Collectively, these data indicate that RBFOX2-mediated regulation of tandem-APA events impact both 3'UTR length and gene expression but also uncovered an enrichment of target genes involved in mitochondrial function.

\section{RBFOX2-mediated tandem-APA regulates 3'UTR length and protein levels of mitochondrial gene SIc25a4 that encodes for ATPIADP translocase 1 (ANT1)}

To better understand how RBFOX2 impacts mitochondrial gene expression, we analyzed mitochondrial genes that displayed both tandem-APA and mRNA level changes and are identified in our GO analysis to be important for muscle development (Supp. Data 
File 3). Our DPAC analysis revealed that total S/c25a4 mRNA levels are downregulated by 1.63 -fold in RBFOX2 depleted cells (Supp. Data File 8). S/c25a4 gene is the ATP/ADP translocator 1 (ANT1) critical for energy production in the mitochondria. It has been shown that ablation of S/c25a4 (ANT1) in mice causes cardiomyopathy associated with defects in mitochondrial respiration (Graham et al., 1997). Loss of function mutations in S/c25a4 are linked to hypertrophic cardiomyopathy and skeletal muscle myopathy in humans (King et al., 2018; Korver-Keularts et al., 2015).

PAC-seq identified a tandem-APA change in SIc25a4 in RBFOX2 KD cells (Supp. Data File 1). S/c25a4 has 2 major PASs (distal and proximal) in its $3^{\prime}$ UTR (Fig. 5A). While distal PAS (dPAS) was minimally used in control cells, its usage was favored in RBFOX2 $K D$ cells (Fig. 4A). This result from PAC-seq was consistent with the nanopore sequencing data that identified mostly SIc25a4 transcripts with long $3^{`}$ UTR generated via dPAS usage in RBFOX2 KD H9c2 cells (Fig. 4B, bottom panel). These results were reproducible in 3 different control and RBFOX2 KD myoblasts (Supp. Fig. 5).

To validate the APA changes in S/c25a4 transcripts, we designed primers to detect long S/c25a4 transcripts generated via dPAS usage and total S/c25a4 transcripts (long and short) (Supp. Table 2). Relative SIc25a4 dPAS/total mRNA ratio was significantly increased in RBFOX2 KD cells (Fig. 4C), consistent with the PAC-seq and nanopore sequencing data indicating increased dPAS usage (Fig. 4A and 4B). Because increased dPAS usage generates S/c25a4 transcripts with long 3'UTRs, which can negatively impact mRNA stability and/or translation, we checked S/c25a4 protein (ANT1) levels. We found that ANT1 protein levels were significantly downregulated in RBFOX2 KD myoblasts (Fig. 4D and 4E), correlating well with the presence of S/c24a5 transcripts with 
long 3'UTR (Fig. 4B and 4C). These results indicate that RBFOX2 is critical for SIc25a4 expression.

RBFOX2 binding motifs near PAS sites are critical for APA-mediated regulation of Slc25a4 expression

To better understand how RBFOX2 regulates SIc25a4 expression via APA, we examined 3'UTR of rat SIc25a4 and found two RBFOX2 binding consensus binding sites one 43nt and another 79nt away from dPAS of S/c25a4 (Fig. 5A). To test whether RBFOX2 binding motifs near the dPAS are functionally important for dPAS usage and S/c25a4 expression, we generated Luciferase-SIc25a4 3'UTR polyA heterologous reporters. While generating these constructs we inserted part of the SIc25a4 3'UTR that contains pPAS, dPAS and RBFOX2 binding sites, downstream of the firefly luciferase open reading frame. We also removed the SV40 PAS within the plasmid (Fig. 5A). In this way, luciferase mRNA will be cleaved and polyadenylated using the PASs within the SIc25a4 3'UTR.

Using this luciferase reporter, we mutated the two RBFOX2 consensus "TGCATG" motifs (Fig. 5A, in red and underlined) near the distal PAS that has been shown to abrogate RBFOX2 binding (Lovci et al., 2013). We tested the relative dPAS usage as well as luciferase activity of the WT construct and compared it to the mutant counterpart that disrupted the RBFOX2 binding site (Fig. 5A, mutant sequence underlined). Mutating RBFOX2 binding motifs in Luciferase-Sc/25a4 3'UTR polyA reporter resulted in a significant increase in dPAS usage (Fig. 5B, WT vs MUT) and a decrease in firefly luciferase activity (Fig. 5C, WT vs MUT). Firefly luciferase activity was downregulated in 
RBFOX2 binding site mutants correlating with increased usage of dPAS generating a longer 3'UTR (Fig. 5B), which can reduce mRNA stability and/or translation. These results (Fig. 5B and 5C) are in agreement with the results from PAC-seq, nanopore reads, RT-PCR validations and by Western blot in RBFOX2 depleted myoblasts (Fig. 4). Altogether, these results suggest that RBFOX2 binding sites close to dPAS repress dPAS usage and modulate SIc25a4 expression levels.

\section{RBFOX2 depletion adversely impacts mitochondrial health}

Since loss of Slc25a4 (ANT1) is linked to cardiomyopathy and skeletal muscle myopathy and mitochondrial defects and Slc25a4 (ANT1) protein levels are downregulated in RBFOX2 KD myoblasts, we investigated the status of mitochondria in RBFOX2 depleted cells. Mitochondrial function is essential for heart and muscle function and it declines in human heart failure and diabetic hearts (Rosca and Hoppel, 2013), in which RBFOX2 is implicated. In addition, mitochondrial defects have been identified in hypoplastic left heart syndrome (Karamanlidis et al., 2011; Liu et al., 2017) in which RBFOX2 mutations have been identified (Homsy et al., 2015; Verma et al., 2016b). The role of RBFOX2 in mitochondrial health is unclear.

Mitochondrial membrane potential is important for respiration and mitochondrion viability (Zorova et al., 2018). This process is affected in RBFOX2 depleted myoblasts (Supp. Data File 9). Therefore, we tested if RBFOX2 KD alters the mitochondrial membrane potential $\left(\Psi_{\mathrm{m}}\right)$, evaluated by the JC-1 assay (Perelman et al., 2012). Mitochondrial membrane potential was increased by 2.5-fold in RBFOX2 KD (vs. control) cells (Fig. 6A). We also used inhibitors of different complexes in the mitochondria to 
assess mitochondrial membrane changes. In response to rotenone (inhibits complex I), antimycin A (inhibits complex III), FCCP (mitochondrial oxidative phosphorylation uncoupler, depolarizes mitochondrial membrane potential), and $\mathrm{H}_{2} \mathrm{O}_{2}$ treatment, mitochondrial membrane potential was downregulated in control cells as expected. This was also the case in RBFOX2 KD cells; however, RBFOX2 KD cells maintained a higher baseline level of $\Psi_{\mathrm{m}}$ than was noted in control cells (Fig. 6A). These results may suggest that increased mitochondrial membrane potential upon RBFOX2 deficiency could be to avoid oxidative injury.

Mitochondrial fusion and fission play a key role in maintaining mitochondrial health. Mitofusin 1 (MFN1) is an outer membrane GTPase that mediates mitochondrial clustering and fusion. Dynamin like OPA1 protein regulates mitochondrial fusion and cristae structure in the inner mitochondrial membrane (Song et al., 2009). These two proteins together regulate the $\Psi_{\mathrm{m}}$ and electron transport chain function and were used as markers to assess electron transport chain function. Our immunofluorescence data showed that RBFOX2 depletion did not overtly impact MFN1 expression but caused a clear decrease in OPA1 levels (Fig. 6B and 6C). Decreased expression of OPA1 protein was consistent with downregulation of its mRNA levels (1.2-fold) in RBFOX2 depleted H9c2 cells (Supp. Data 2). To further determine the effect of RBFOX2 on mitochondrial health, we performed transmission electron microscopy to assess mitochondrial ultrastructure and morphology. We found that in RBFOX2 KD H9c2 cells, mitochondria were swollen as seen in representative micrographs (Fig. 6D, black arrows indicate mitochondria) and also evident from increased mitochondrial area in RBFOX2-depleted cells (Fig. 6E). These type of morphological changes in mitochondria have been linked to the rupture of 
mitochondria and apoptosis. Our findings indicate that RBFOX2 is important for mitochondrial health, which is relevant to its role in heart diseases.

\section{DISCUSSION}

RBFOX2 is implicated in cardiovascular diseases, congenital heart defects and skeletal muscle development (Gallagher et al., 2011; Homsy et al., 2015; Nutter et al., 2016b; Singh et al., 2018; Singh et al., 2014; Verma et al., 2016b; Wei et al., 2015a). However, it is not clear whether RBFOX2 has additional roles other than AS regulation that influences gene expression and function in muscle cells. In this study, we addressed this fundamentally important question utilizing state of the art techniques and performing functional assays. We uncovered a critical new role for RBFOX2 in maintaining APA patterns in myoblasts and provided insights into the functional consequences of APA changes mediated by RBFOX2 in myoblasts. In addition, our results revealed a novel role for RBFOX2 in regulating mitochondrial health relevant to human heart diseases.

In this study, combined usage of PAC-seq and nanopore sequencing allowed us to precisely determine RBFOX2-regulated APA patterns and evaluate the consequences of RBFOX2-mediated APA changes. PAC-seq allowed us to sensitively detect poly(A)sites and simultaneously measure transcript abundance, while nanopore sequencing with MinION allowed us to validate PAS usage, simultaneously characterize exon inclusion across the entire mRNA transcript and determine 3'UTR length. By combining PAC-seq and nanopore sequencing, we discovered a new role for RBFOX2 in APA regulation. We identified 233 APA changes in RBFOX2 KD myoblasts and found that $46 \%$ of these APA changes impacted mRNA levels. APA regulation is linked to physiological 
processes including heart development (Nimura et al., 2016), cell proliferation in immune response (Sandberg et al., 2008), and embryonic and postnatal development (Mangone et al., 2010). Aberrant regulation of APA is linked to pathological conditions including heart failure, cancer and muscular dystrophy (Batra et al., 2014; Creemers et al., 2016; Masamha et al., 2014). Here, we showed that RBFOX2-dependent APA events impacted essential contractile gene Tropomyosin 1. By identifying developmentally regulated APA networks that impact muscle-specific gene expression and gene isoforms, we provide insights into the post-transcriptional regulation of these genes in the developing muscle.

AS can directly affect PAS usage and APA by regulating the splicing of exons that harbor PASs. RBFOX2 is a well-known regulator of AS. Despite its prominent role in AS, the majority of RBFOX2 induced APA changes were not mediated via splicing-APA. Instead, it was mediated via tandem-APA, which affected the length of 3'UTRs. We validated RBFOX2-mediated splicing-APA and tandem-APA using several different methods including PAC-seq, nanopore sequencing and RT-PCR. We identified rat Slc25a4 gene as a target of RBFOX2 and found that RBFOX2 binding sites near PASs are important determinants of Slc25a4 dPAS usage and its expression levels. Mutating RBFOX2 binding motifs close to the dPAS increased dPAS usage, suggesting that RBFOX2 may act as a repressor of PAS usage when bound nearby.

In this study, we also focused on the functional consequences of RBFOX2mediated APA and gene expression changes. We found that RBFOX2 is critical in maintaining mitochondrial gene expression (i.e OPA1 and Slc25a4) and mitochondrial health. Importantly, we identified SIc25a4 gene which encodes for ANT1 protein as a new 
APA target of RBFOX2. ANT1 is necessary for ATP/ADP translocation across the mitochondrial membrane during oxidative phosphorylation (King et al., 2018; KorverKeularts et al., 2015). Mutations in this gene is linked to cardiomyopathy in human patients (King et al., 2018; Korver-Keularts et al., 2015). We found that RBFOX2 depletion affected SIc25a4 APA, and its expression levels. Consistently, both Rbfox2 deletion in cardiomyocytes and loss of S/c25a4 function resulted in dilated cardiomyopathy (Wei et al., 2015a) (King et al., 2018; Korver-Keularts et al., 2015). Mitochondrial membrane potential is important for ATP production, which is required for heart and muscle function and reduced mitochondrial function is a hallmark of failing hearts (Rosca and Hoppel, 2013). Importantly, we found profound changes in mitochondrial health upon RBFOX2 depletion. Our findings demonstrate that RBFOX2 controls mitochondrial gene expression via APA, providing novel insights into how RBFOX2 may alter mitochondrial health in diseased hearts. 


\section{ACCESSION NUMBERS}

All raw sequencing data generated by Nanopore sequencing and PAC-Seq in this manuscript are deposited into the NCBI SRA database with project number PRJNA517125.

\section{ACKNOWLEDGEMENTS}

The authors acknowledge the University of Texas Medical Branch Next Generation Sequencing Core Facility for providing RNA sequencing services. We thank Dr. Pei-Yong Shi and Dr.Ricardo Rajsbaum for allowing us to use their plate readers.

\section{AUTHOR CONTRIBUTIONS}

M.K.M conceived the idea, conceptualized the project, interpreted the data, provided financial support and wrote the manuscript. A.R designed the DPAC pipeline, processed and analyzed the PAC-seq and nanopore data, provided financial support and critically edited the manuscript drafts. J.C conceptualized the project, designed and performed most of the experiments, analyzed data, wrote the manuscript and confirmed the accuracy of data presented in the manuscript. S.K.V. performed experiments related to S/c25a4 APA, graphed the plots, performed statistics, analyzed the data and provided feedback. S.M. (S. Mohan) performed the analysis for endogenous S/c25a4 mRNA and protein levels. S.N.M and A.S helped to clone polyA reporters. C.N performed some of the S/c25a4 RT-PCRs. R.J.H. designed and optimized primers and performed some of the cDNA synthesis. E.J. performed the nanopore sequencing. P.L generated some of 
the PAC-seq libraries. N.E helped with the preliminary analysis of the PAC-seq data. K.R designed and performed all experiments and analyzed data related to mitochondrial studies. V.P prepared the samples and processed them for TEM and helped analyze the data. E.J.W critically analyzed/interpreted the data and provided feedback for the design of experiments and edited the manuscript drafts. E.Y. provided feedback for the organization of the manuscript and design of the experiments. N.J.G provided financial support for carrying out the mitochondrial function related experiments and helped write and edit the manuscript drafts. All authors have read and approved the manuscript.

\section{SOURCES OF FUNDING}

This work was supported, in part, UTMB Department of Biochemistry and Molecular Biology Bridging funds; and grants from the National Institutes of Health/ National Heart Lung Blood Institute [1R01HL135031] and American Heart Association [20TPA35490206] to M.N.K-M. The contents of the manuscript are solely the responsibility of the authors and do not necessarily represent the official views of NHLBI of NIH. J.C. is a funded by a post-doctoral fellowship from American Heart Association [18POST3399018]. N.J.G. is funded by grants from the National Institute of Allergy and Infectious Diseases (R01Al054578; R01Al136031) of the National Institutes of Health. A.R. is supported by start-up funds from UTMB. E.J.W. acknowledges support of BMB Startup funds and P.J is supported by funds from the National Institutes of Health (R03CA223893).

\section{DECLARATION OF INTERESTS}

Authors declare no conflict of interest. 


\section{REFERENCES}

Batra, R., Charizanis, K., Manchanda, M., Mohan, A., Li, M., Finn, D.J., Goodwin, M., Zhang, C., Sobczak, K., Thornton, C.A., et al. (2014). Loss of MBNL leads to disruption of developmentally regulated alternative polyadenylation in RNA-mediated disease. Molecular cell 56, 311-322.

Belanger, K., Nutter, C.A., Li, J., Tasnim, S., Liu, P.R., Yu, P., and Kuyumcu-Martinez, M.N. (2018). CELF1 contributes to aberrant alternative splicing patterns in the type 1 diabetic heart. Biochem Bioph Res Co 503, 3205-3211.

Cooke, C., Hans, H., and Alwine, J.C. (1999). Utilization of splicing elements and polyadenylation signal elements in the coupling of polyadenylation and last-intron removal. Mol Cell Biol 19, 4971-4979.

Creemers, E.E., Bawazeer, A., Ugalde, A.P., van Deutekom, H.W., van der Made, I., de Groot, N.E., Adriaens, M.E., Cook, S.A., Bezzina, C.R., Hubner, N., et al. (2016). Genome-Wide Polyadenylation Maps Reveal Dynamic mRNA 3'-End Formation in the Failing Human Heart. Circulation research 118, 433-438.

Damianov, A., Ying, Y., Lin, C.H., Lee, J.A., Tran, D., Vashisht, A.A., Bahrami-Samani, E., Xing, Y., Martin, K.C., Wohlschlegel, J.A., et al. (2016). Rbfox Proteins Regulate Splicing as Part of a Large Multiprotein Complex LASR. Cell 165, 606-619.

Derti, A., Garrett-Engele, P., Macisaac, K.D., Stevens, R.C., Sriram, S., Chen, R., Rohl, C.A., Johnson, J.M., and Babak, T. (2012). A quantitative atlas of polyadenylation in five mammals. Genome Res 22, 1173-1183.

Di Giammartino, D.C., Nishida, K., and Manley, J.L. (2011). Mechanisms and consequences of alternative polyadenylation. Molecular cell 43, 853-866. 
England, J., Granados-Riveron, J., Polo-Parada, L., Kuriakose, D., Moore, C., Brook, J.D., Rutland, C.S., Setchfield, K., Gell, C., Ghosh, T.K., et al. (2017). Tropomyosin 1: Multiple roles in the developing heart and in the formation of congenital heart defects. Journal of molecular and cellular cardiology 106, 1-13.

Gallagher, T.L., Arribere, J.A., Geurts, P.A., Exner, C.R.T., McDonald, K.L., Dill, K.K., Marr, H.L., Adkar, S.S., Garnett, A.T., Amacher, S.L., et al. (2011). Rbfox-regulated alternative splicing is critical for zebrafish cardiac and skeletal muscle functions. Dev Biol $359,251-261$.

Gooding, C., and Smith, C.W.J. (2008). Tropomyosin Exons as Models for Alternative Splicing. Adv Exp Med Biol 644, 27-42.

Graham, B.H., Waymire, K.G., Cottrell, B., Trounce, I.A., MacGregor, G.R., and Wallace, D.C. (1997). A mouse model for mitochondrial myopathy and cardiomyopathy resulting from a deficiency in the heart/muscle isoform of the adenine nucleotide translocator. Nat Genet 16, 226-234.

Homsy, J., Zaidi, S., Shen, Y., Ware, J.S., Samocha, K.E., Karczewski, K.J., DePalma, S.R., McKean, D., Wakimoto, H., Gorham, J., et al. (2015). De novo mutations in congenital heart disease with neurodevelopmental and other congenital anomalies. Science 350, 1262-1266.

Huang, S.C., Ou, A.C., Park, J., Yu, F., Yu, B., Lee, A., Yang, G., Zhou, A., and Benz, E.J., Jr. (2012). RBFOX2 promotes protein 4.1R exon 16 selection via U1 snRNP recruitment. Mol Cell Biol 32, 513-526. 
Ignatiadis, N., Klaus, B., Zaugg, J.B., and Huber, W. (2016). Data-driven hypothesis weighting increases detection power in genome-scale multiple testing. Nat Methods 13, 577-580.

Karamanlidis, G., Bautista-Hernandez, V., Fynn-Thompson, F., Del Nido, P., and Tian, R. (2011). Impaired mitochondrial biogenesis precedes heart failure in right ventricular hypertrophy in congenital heart disease. Circ Heart Fail 4, 707-713.

King, M.S., Thompson, K., Hopton, S., He, L., Kunji, E.R.S., Taylor, R.W., and OrtizGonzalez, X.R. (2018). Expanding the phenotype of de novo SLC25A4-linked mitochondrial disease to include mild myopathy. Neurol Genet 4, e256.

Korver-Keularts, I.M., de Visser, M., Bakker, H.D., Wanders, R.J., Vansenne, F., Scholte, H.R., Dorland, L., Nicolaes, G.A., Spaapen, L.M., Smeets, H.J., et al. (2015). Two Novel Mutations in the SLC25A4 Gene in a Patient with Mitochondrial Myopathy. JIMD Rep 22, $39-45$.

Kuleshov, M.V., Jones, M.R., Rouillard, A.D., Fernandez, N.F., Duan, Q., Wang, Z., Koplev, S., Jenkins, S.L., Jagodnik, K.M., Lachmann, A., et al. (2016). Enrichr: a comprehensive gene set enrichment analysis web server 2016 update. Nucleic acids research 44 , W90-97.

Li, H. (2016). Minimap and miniasm: fast mapping and de novo assembly for noisy long sequences. Bioinformatics 32, 2103-2110.

Li, H. (2018). Minimap2: pairwise alignment for nucleotide sequences. Bioinformatics 34 , 3094-3100.

Lin, J.J.C., Eppinga, R.D., Warren, K.S., and McCrae, K.R. (2008). Human Tropomyosin Isoforms in the Regulation of Cytoskeleton Functions. Adv Exp Med Biol 644, 201-222. 
Liu, X., Yagi, H., Saeed, S., Bais, A.S., Gabriel, G.C., Chen, Z., Peterson, K.A., Li, Y., Schwartz, M.C., Reynolds, W.T., et al. (2017). The complex genetics of hypoplastic left heart syndrome. Nat Genet 49, 1152-1159.

Lovci, M.T., Ghanem, D., Marr, H., Arnold, J., Gee, S., Parra, M., Liang, T.Y., Stark, T.J., Gehman, L.T., Hoon, S., et al. (2013). Rbfox proteins regulate alternative mRNA splicing through evolutionarily conserved RNA bridges. Nature structural \& molecular biology 20, 1434-1442.

Love, M.I., Huber, W., and Anders, S. (2014). Moderated estimation of fold change and dispersion for RNA-seq data with DESeq2. Genome Biol 15, 550.

Mangone, M., Manoharan, A.P., Thierry-Mieg, D., Thierry-Mieg, J., Han, T., Mackowiak, S.D., Mis, E., Zegar, C., Gutwein, M.R., Khivansara, V., et al. (2010). The landscape of C. elegans 3'UTRs. Science 329, 432-435.

Masamha, C.P., Xia, Z., Yang, J., Albrecht, T.R., Li, M., Shyu, A.B., Li, W., and Wagner, E.J. (2014). CFIm25 links alternative polyadenylation to glioblastoma tumour suppression. Nature 510, 412-416.

Misra, C., Bangru, S., Lin, F., Lam, K., Koenig, S.N., Lubbers, E.R., Hedhli, J., Murphy, N.P., Parker, D.J., Dobrucki, L.W., et al. (2020). Aberrant Expression of a Non-muscle RBFOX2 Isoform Triggers Cardiac Conduction Defects in Myotonic Dystrophy. Dev Cell 52, 748-763 e746.

Nimura, K., Yamamoto, M., Takeichi, M., Saga, K., Takaoka, K., Kawamura, N., Nitta, H., Nagano, H., Ishino, S., Tanaka, T., et al. (2016). Regulation of alternative polyadenylation by Nkx2-5 and Xrn2 during mouse heart development. eLife 5. 
Nutter, C.A., Jaworski, E., Verma, S.K., Perez-Carrasco, Y., and Kuyumcu-Martinez, M.N. (2017). Developmentally Regulated Alternative Splicing Is Perturbed in Type 1 Diabetic Skeletal Muscle. Muscle Nerve 56, 744-749.

Nutter, C.A., Jaworski, E.A., Verma, S.K., Deshmukh, V., Wang, Q., Botvinnik, O.B., Lozano, M.J., Abass, I.J., Ijaz, T., Brasier, A.R., et al. (2016a). Dysregulation of RBFOX2 Is an Early Event in Cardiac Pathogenesis of Diabetes. Cell Rep 15, 2200-2213.

Nutter, C.A., Jaworski, E.A., Verma, S.K., Deshmukh, V., Wang, Q.L., Botvinnik, O.B., Lozano, M.J., Abass, I.J., ljaz, T., Brasier, A.R., et al. (2016b). Dysregulation of RBFOX2 Is an Early Event in Cardiac Pathogenesis of Diabetes. Cell Rep 15, 2200-2213.

Perelman, A., Wachtel, C., Cohen, M., Haupt, S., Shapiro, H., and Tzur, A. (2012). JC-1: alternative excitation wavelengths facilitate mitochondrial membrane potential cytometry. Cell Death Dis 3, e430.

Rosca, M.G., and Hoppel, C.L. (2013). Mitochondrial dysfunction in heart failure. Heart failure reviews $18,607-622$.

Routh, A. (2019a). DPAC: A Tool for Differential Poly(A)-Cluster Usage from Poly(A)Targeted RNAseq Data. G3 9, 1825-1830.

Routh, A., Ji, P., Jaworski, E., Xia, Z., Li, W., and Wagner, E.J. (2017). Poly(A)-ClickSeq: click-chemistry for next-generation 3-end sequencing without RNA enrichment or fragmentation. Nucleic acids research 45, e112.

Routh, A.L. (2019b). DPAC: a tool for Differential Poly(A) Site usage from poly(A) targeted RNAseq data. bioRxiv, 531590. 
Runfola, V., Sebastian, S., Dilworth, F.J., and Gabellini, D. (2015). Rbfox proteins regulate tissue-specific alternative splicing of Mef2D required for muscle differentiation. Journal of cell science $128,631-637$.

Sandberg, R., Neilson, J.R., Sarma, A., Sharp, P.A., and Burge, C.B. (2008). Proliferating cells express mRNAs with shortened $3^{\prime}$ untranslated regions and fewer microRNA target sites. Science 320, 1643-1647.

Shi, Y. (2012). Alternative polyadenylation: new insights from global analyses. Rna 18, 2105-2117.

Singh, R.K., Kolonin, A.M., Fiorotto, M.L., and Cooper, T.A. (2018). Rbfox-Splicing Factors Maintain Skeletal Muscle Mass by Regulating Calpain3 and Proteostasis. Cell Rep 24, 197-208.

Singh, R.K., Xia, Z., Bland, C.S., Kalsotra, A., Scavuzzo, M.A., Curk, T., Ule, J., Li, W., and Cooper, T.A. (2014). Rbfox2-coordinated alternative splicing of Mef2d and Rock2 controls myoblast fusion during myogenesis. Molecular cell 55, 592-603.

Song, Z., Ghochani, M., McCaffery, J.M., Frey, T.G., and Chan, D.C. (2009). Mitofusins and OPA1 mediate sequential steps in mitochondrial membrane fusion. Molecular biology of the cell $20,3525-3532$.

Sun, S., Zhang, Z., Fregoso, O., and Krainer, A.R. (2012). Mechanisms of activation and repression by the alternative splicing factors RBFOX1/2. Rna 18, 274-283.

Tang, A.D., Soulette, C.M., Baren, M.J.v., Hart, K., Hrabeta-Robinson, E., Wu, C.J., and Brooks, A.N. (2018). Full-length transcript characterization of $<$ em $>$ SF3B1</em> mutation in chronic lymphocytic leukemia reveals downregulation of retained introns. bioRxiv, 410183. 
Thomas, A., Rajan, S., Thurston, H.L., Masineni, S.N., Dube, P., Bose, A., Muthu, V., Dube, S., Wieczorek, D.F., Poiesz, B.J., et al. (2010). Expression of a novel tropomyosin isoform in axolotl heart and skeletal muscle. Journal of cellular biochemistry $110,875-$ 881.

Tian, B., Hu, J., Zhang, H., and Lutz, C.S. (2005). A large-scale analysis of mRNA polyadenylation of human and mouse genes. Nucleic acids research 33, 201-212.

Verma, S.K., Deshmukh, V., Liu, P., Nutter, C.A., Espejo, R., Hung, M.L., Wang, G.S., Yeo, G.W., and Kuyumcu-Martinez, M.N. (2013). Reactivation of fetal splicing programs in diabetic hearts is mediated by protein kinase $C$ signaling. The Journal of biological chemistry $288,35372-35386$.

Verma, S.K., Deshmukh, V., Nutter, C.A., Jaworski, E., Jin, W., Wadhwa, L., Abata, J., Ricci, M., Lincoln, J., Martin, J.F., et al. (2016a). Rbfox2 function in RNA metabolism is impaired in hypoplastic left heart syndrome patient hearts. Sci Rep 6, 30896.

Verma, S.K., Deshmukh, V., Nutter, C.A., Jaworski, E., Jin, W.H., Wadhwa, L., Abata, J., Ricci, M., Lincoln, J., Martin, J.F., et al. (2016b). Rbfox2 function in RNA metabolism is impaired in hypoplastic left heart syndrome patient hearts. Sci Rep-Uk 6.

Wang, E.T., Sandberg, R., Luo, S., Khrebtukova, I., Zhang, L., Mayr, C., Kingsmore, S.F., Schroth, G.P., and Burge, C.B. (2008). Alternative isoform regulation in human tissue transcriptomes. Nature 456, 470-476.

Wei, C., Qiu, J., Zhou, Y., Xue, Y., Hu, J., Ouyang, K., Banerjee, I., Zhang, C., Chen, B., Li, H., et al. (2015a). Repression of the Central Splicing Regulator RBFox2 Is Functionally Linked to Pressure Overload-Induced Heart Failure. Cell Rep 10, 1521-1533. 
Wei, C.L., Quu, J.S., Zhou, Y., Xue, Y.C., Hu, J., Ouyang, K.F., Banerjee, I., Zhang, C.M., Chen, B.Y., Li, H.R., et al. (2015b). Repression of the Central Splicing Regulator RBFox2 Is Functionally Linked to Pressure Overload-Induced Heart Failure. Cell Rep 10, 15211533.

Weyn-Vanhentenryck, S.M., Mele, A., Yan, Q., Sun, S., Farny, N., Zhang, Z., Xue, C., Herre, M., Silver, P.A., Zhang, M.Q., et al. (2014). HITS-CLIP and integrative modeling define the Rbfox splicing-regulatory network linked to brain development and autism. Cell Rep 6, 1139-1152.

Wolska, B.M., and Wieczorek, D.M. (2003). The role of tropomyosin in the regulation of myocardial contraction and relaxation. Pflugers Archiv : European journal of physiology $446,1-8$.

Yeo, G.W., Coufal, N.G., Liang, T.Y., Peng, G.E., Fu, X.D., and Gage, F.H. (2009). An RNA code for the FOX2 splicing regulator revealed by mapping RNA-protein interactions in stem cells. Nature structural \& molecular biology 16, 130-137.

Zorova, L.D., Popkov, V.A., Plotnikov, E.Y., Silachev, D.N., Pevzner, I.B., Jankauskas, S.S., Babenko, V.A., Zorov, S.D., Balakireva, A.V., Juhaszova, M., et al. (2018). Mitochondrial membrane potential. Analytical biochemistry 552, 50-59. 


\section{FIGURES AND FIGURE LEGENDS}

Figure 1. RBFOX2 depletion in $\mathrm{H} 9 \mathrm{c} 2$ myoblasts leads to altered alternative polyadenylation patterns determined by Poly(A)-ClickSeq (PAC-seq).

(A) A representative Western blot showing efficient knock down (KD) of RBFOX2 in H9c2 myoblasts. Ponceau stained membrane was used to monitor protein loading in each lane.

(B) The number of genes undergo tandem-APA or splicing-APA in RBFOX2 KD H9c2 myoblasts.

(C) Gene ontology (GO) analysis of genes that undergo APA changes in RBFOX2 depleted myoblasts. 


\section{Figure 1}

A

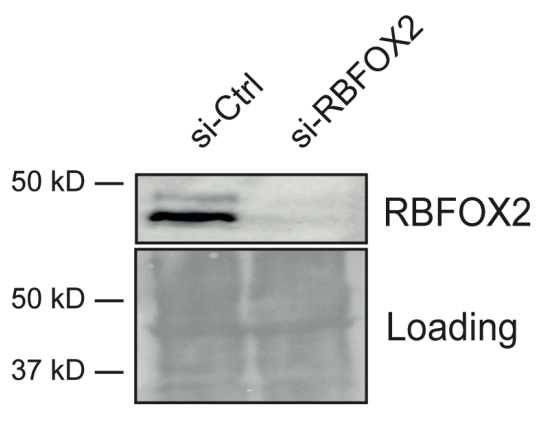

B

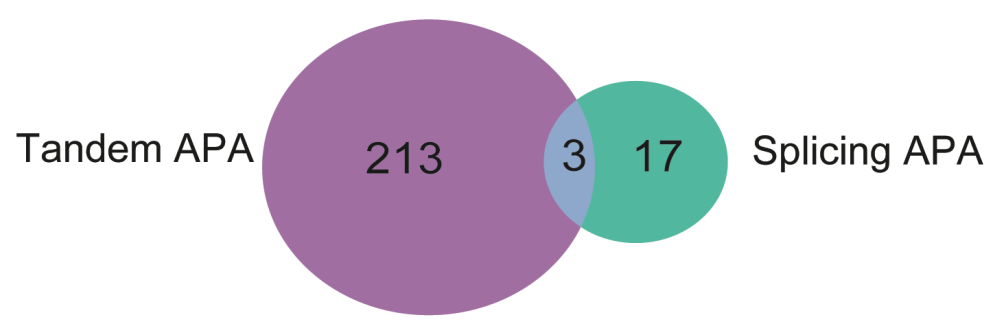

C

Biological processes affected via APA (DAVID)

\begin{tabular}{|l|c|c|}
\hline \hline & $\begin{array}{c}\text { Adjusted } \\
\mathrm{p} \text {-value }\end{array}$ & $\begin{array}{c}\text { Number of } \\
\text { genes }\end{array}$ \\
\hline $\begin{array}{l}\text { Regulation of cellular component } \\
\text { organization }\end{array}$ & 0.038 & 50 \\
\hline Musle cell development & 0.029 & 11 \\
\hline Striated muscle cell development & 0.041 & 11 \\
\hline
\end{tabular}


Figure 2. RBFOX2 regulates expression levels of muscle-specific isoforms of rat Tropomyosin 1 (Tpm1) via splicing-APA in H9c2 myoblasts.

(A) The poly(A) usage of Tpm1 transcripts in control and RBFOX2 depleted H9c2 cells determined by poly(A)-ClickSeq, $(n=3)$. PolyA sites located in either terminal exon 9b or terminal exon 9d were utilized in $\mathrm{H} 9 \mathrm{c} 2$ myoblasts.

(B) Full-length Tpm1 short (muscle) or long (non-muscle) isoforms identified by nanopore sequencing in control vs RBFOX2 depleted H9c2 myoblasts $(n=3)$.

(C) Relative mRNA levels of Tpm1 muscle-specific (short) transcripts that end with exon 9b in control and RBFOX2 depleted H9c2 cells were determined by RT-qPCR. mRNA levels in control cells were normalized to 1 . Data represent means \pm SD. Statistical significance was calculated using t-test to compare two different groups in three independent experiments $(n=3) .{ }^{* *} p=0.0079$.

(D) Relative protein levels of TPM1 in control and RBFOX2 depleted H9c2 cells determined by Western blot. $\alpha$-Tubulin was used as a loading control. Protein levels in control cells were normalized to 1 . Data represent means \pm SD. Statistical significance was calculated using t-test to compare two different groups in three independent experiments $(n=3){ }^{* * *} p=0.0001$.

(E) Relative mRNA levels of Tpm1 non-muscle transcripts (long) in control and RBFOX2 depleted H9c2 cells. mRNA levels in control cells were normalized to 1. Data represent means $\pm S D$. No statistical significance was found using t-test in three independent experiments $(n=3)$. 
(F) RBFOX2 protein expression levels in (1) scrambled siRNA treated, (2) RBFOX2 siRNA treated, (3) RBFOX2 siRNA treated H9c2 cells ectopically expressing GFP or GFP-RBFOX2.

(G) Ectopic expression of GFP-RBFOX2 partially rescued splicing-APA change in Tpm1 caused by RBFOX2 depletion. Relative expression levels of muscle-specific Tpm1 (short) transcripts in scrambled siRNA treated, RBFOX2 siRNA treated, or RBFOX2 siRNA treated H9c2 myoblasts expressing GFP or GFP-RBFOX2. mRNA levels in control cells (1) were normalized to 1 . Data represent means \pm SD. Statistical significance was calculated using one-way ANOVA to compare three different groups in three independent experiments ( $n=3)$. P-value for Control ${ }^{\operatorname{siRNA}}+$ GFP vs. RBFOX2 $2^{\operatorname{siRNA}}+G F P$ is ${ }^{* * *} p<0.0001$; for RBFOX2 ${ }^{\text {IiRNA }_{+}}$GFP vs. RBFOX2 ${ }^{\text {siRNA }}+$ GFP-RBFOX2 is ${ }^{* *} p=0.0056$; for Control ${ }^{\text {iRNA }}+$ GFP vs. RBFOX2 $2^{\text {siRNA }}+$ GFP-RBFOX2 is ${ }^{* *} p=0.0027$.

(H) Relative expression levels of non-muscle Tpm1 (long) transcripts in control, RBFOX2 depleted, or RBFOX2 depleted GFP or GFP-RBFOX2 expressing cells. mRNA levels in (1) cells were normalized to 1 . Data represent means \pm SD. Statistical significance was calculated using one-way ANOVA to compare three different groups in three independent experiments $(n=3)$. 
bioRxiv preprint doi: https://doi.org/10.1101/2020.05.13.093013; this version posted March 25, 2021. The copyright holder for this preprint (which was not certified by peer review) is the author/funder. All rights reserved. No reuse allowed without permission.

\section{Figure 2}

A

3'-end of Rat Tpm1

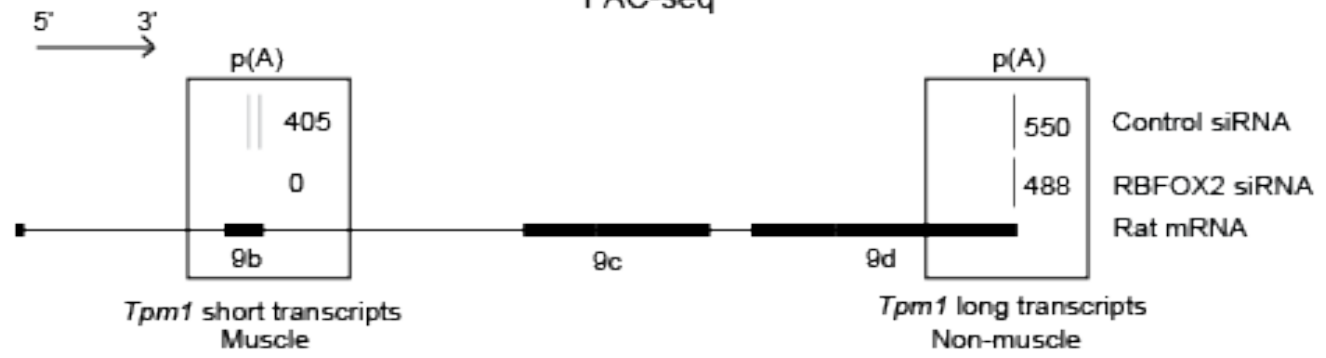

PAC-seq

B

Nanopore-seq

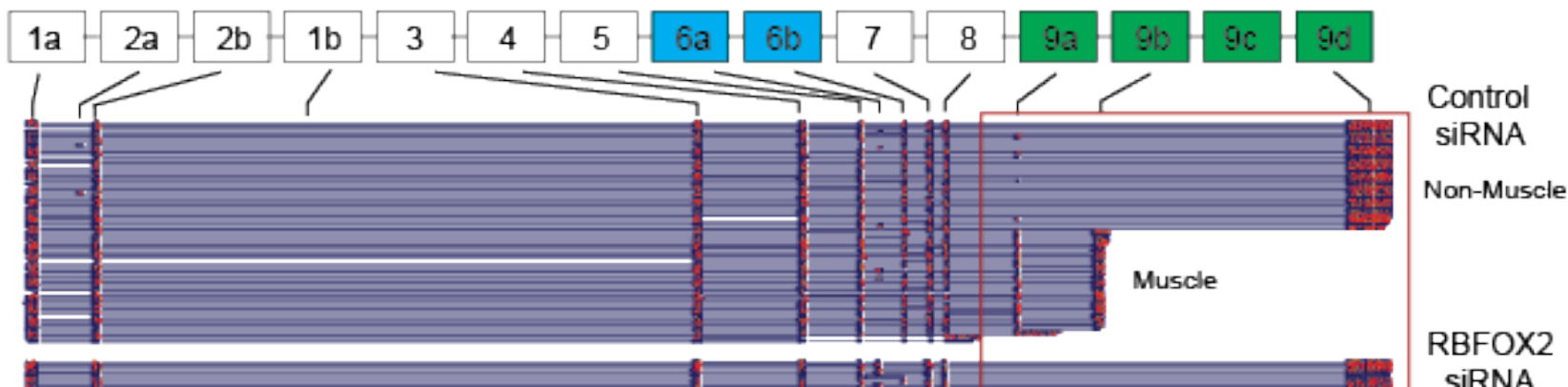

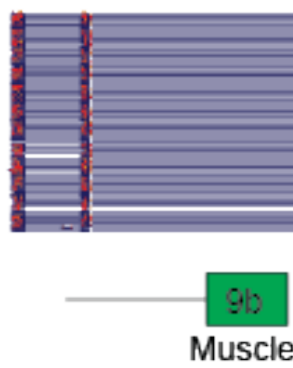

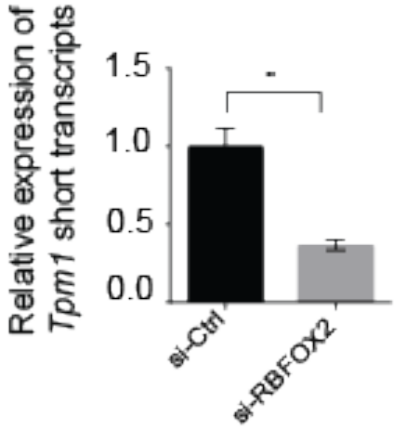

$\mathrm{F}$

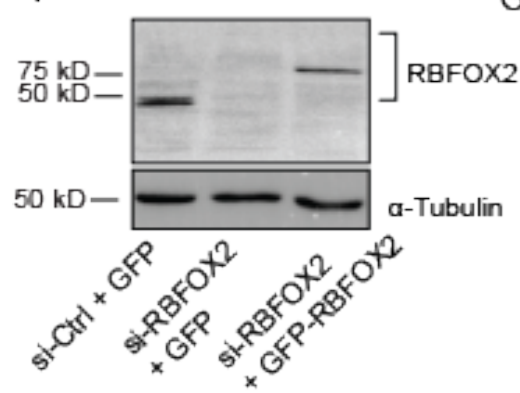

D

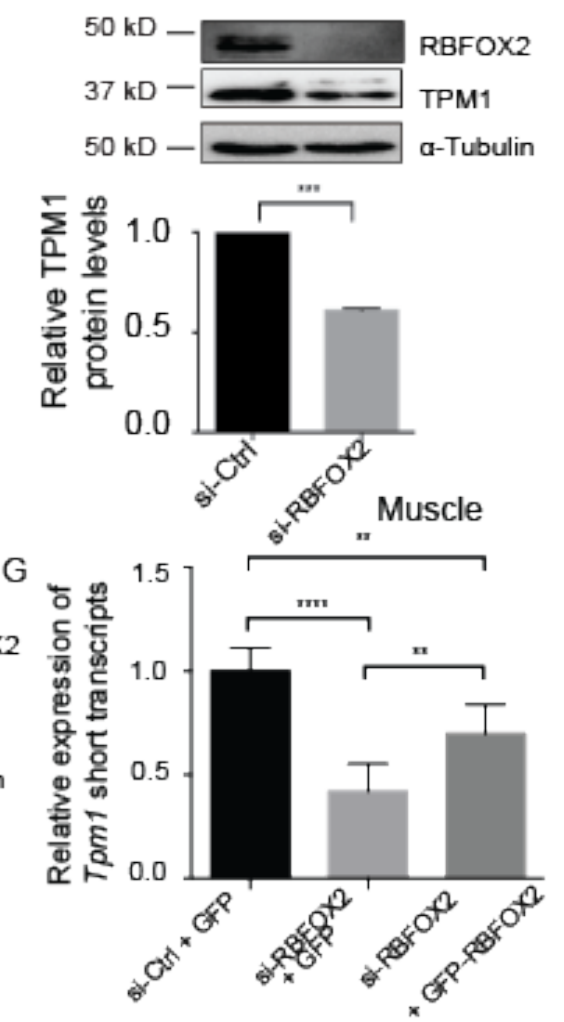

E

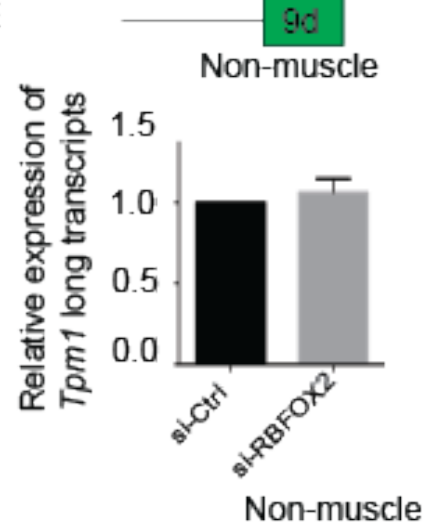

$\mathrm{H}$

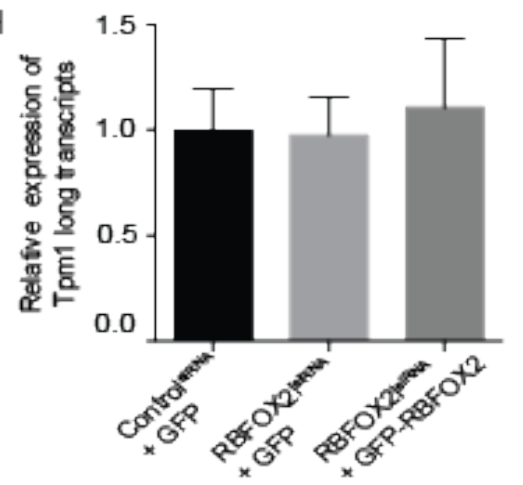


Figure 3. RBFOX2-regulated tandem-APA modulates $3^{\prime} U T R$ length and affects mRNA levels.

(A) The number of genes that undergo tandem-APA changes generating shorter or longer 3'UTRs in RBFOX2 KD H9c2 myoblasts.

(B) Enrichr TargetScan analysis of genes that display 3'UTR length changes upon RBFOX2 depletion revealed known microRNA binding sites within majority of these genes.

(C) Gene expression changes in RBFOX2 KD H9c2 myoblasts.

(D) Gene ontology analysis of genes that are downregulated in RBFOX2 depleted H9c2 myoblasts.

(E) The number of genes that exhibit both APA and gene expression changes in RBFOX2 KD H9c2 myoblasts. 


\section{Figure 3}

A

RBFOX2- dependent

3'UTR 3'UTR

shortening lengthening

$129 \quad 23 \quad 64$

C

RBFOX2- dependent mRNA

level changes

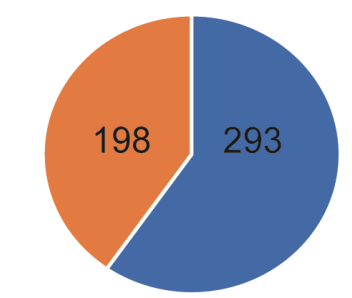

Downregulated genes

- Upregulated genes
B

RBFOX2- dependent APA genes with 3'UTR length changes

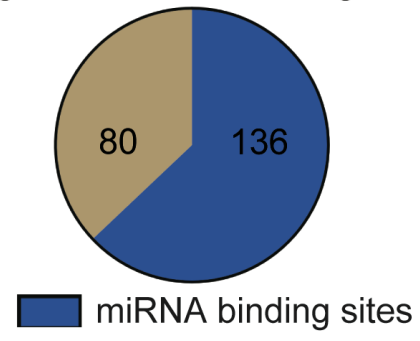

No miRNA binding sites

D

Biological processes affected

\begin{tabular}{|l|c|c|c|}
\hline & $\begin{array}{c}\text { Adjusted } \\
\text { p-value }\end{array}$ & $\begin{array}{c}\text { Combined } \\
\text { score }\end{array}$ & $\begin{array}{c}\text { Number of } \\
\text { genes }\end{array}$ \\
\hline mitochondrial inner membrane & $3.61 \mathrm{e}-13$ & 266 & $26 / 342$ \\
\hline mitochondrion & $7.75 \mathrm{e}-09$ & 85 & $36 / 1027$ \\
\hline $\begin{array}{l}\text { mitochondrial respiratory } \\
\text { chain complex I }\end{array}$ & $1.24 \mathrm{e}-08$ & 449 & $10 / 52$ \\
\hline
\end{tabular}

E

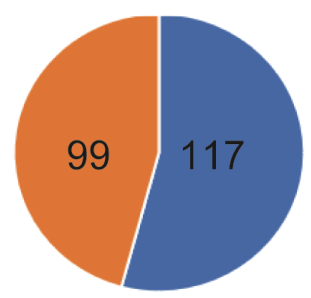

RBFOX2-regulated APA genes with $\mathrm{mRNA}$ level changes

RBFOX2-regulated APA genes without mRNA level changes 
Figure 4. RBFOX2 regulates tandem-APA and expression of essential mitochondrial gene SIc25a4.

(A) SIc25a4 3'UTR lengthening mediated via tandem-APA in RBFOX2 depleted H9c2 myoblasts identified by PAC-seq.

(B) Nanopore sequencing analysis of full length S/c25a4 transcripts with different 3'UTR lengths in control vs RBFOX2 KD myoblasts.

(C) RT-PCR analysis of endogenous S/c25a4 dPAS/total mRNA ratio in RBFOX2 KD myoblasts. $n=8,{ }^{* * * *} p<0.0001$ (three independent experiments).

(D) Western blot analysis of ANT1 protein (SIc25a4) in control vs RBFOX2 KD myoblasts determined by Bio-Rad ChemiDoc Imager. Even protein loading was monitored by imaging stain-free gels. ANT1 protein levels were normalized to loading control and fold change in ANT1 protein levels were quantified using Bio-Rad ChemiDoc software. $n=4$, ${ }^{* * *} p=0.0002$. 


\section{Figure 4}

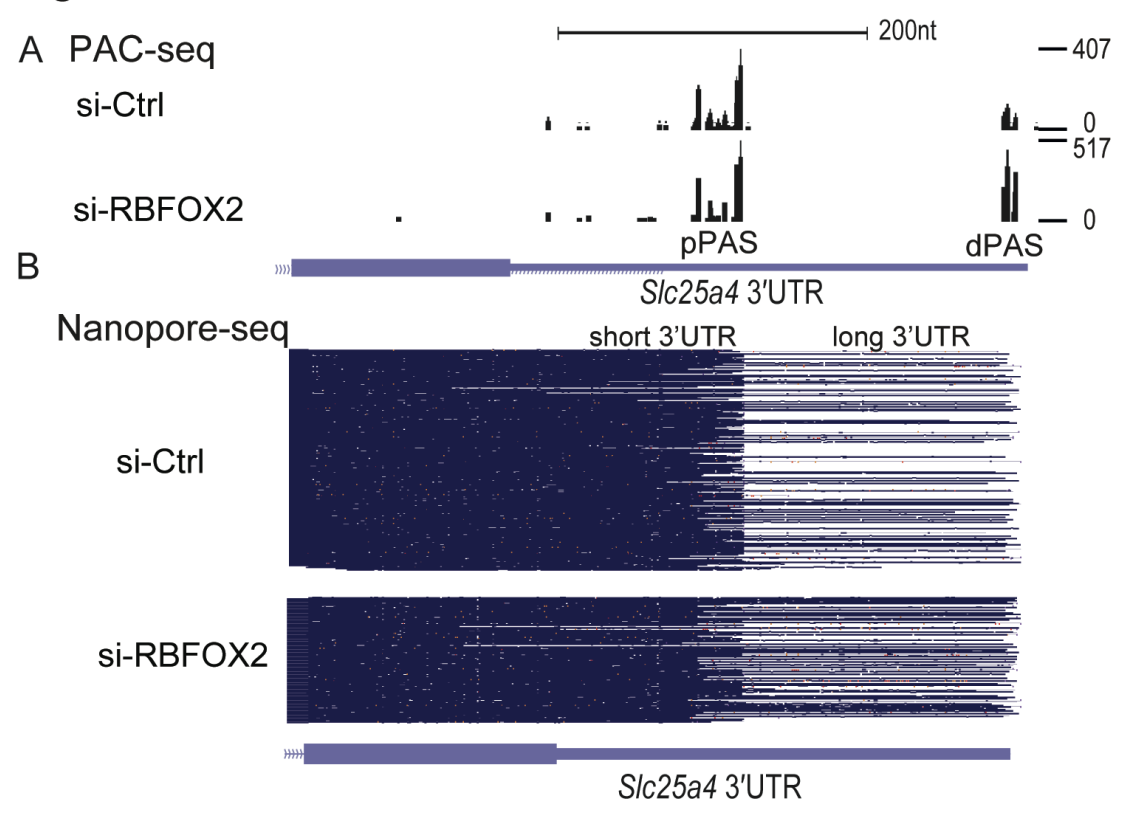

C

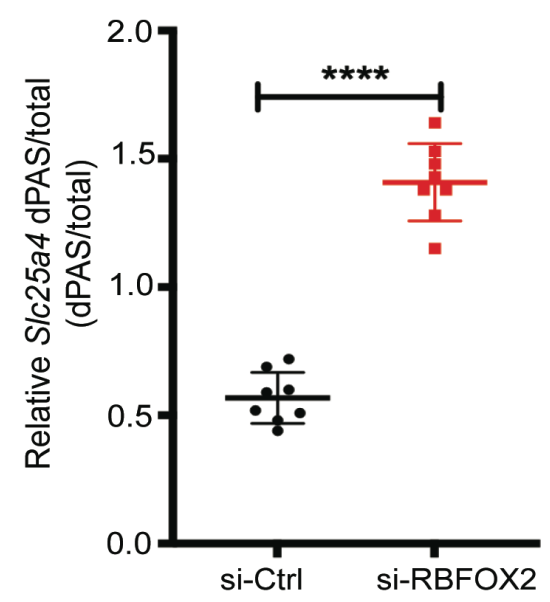

D

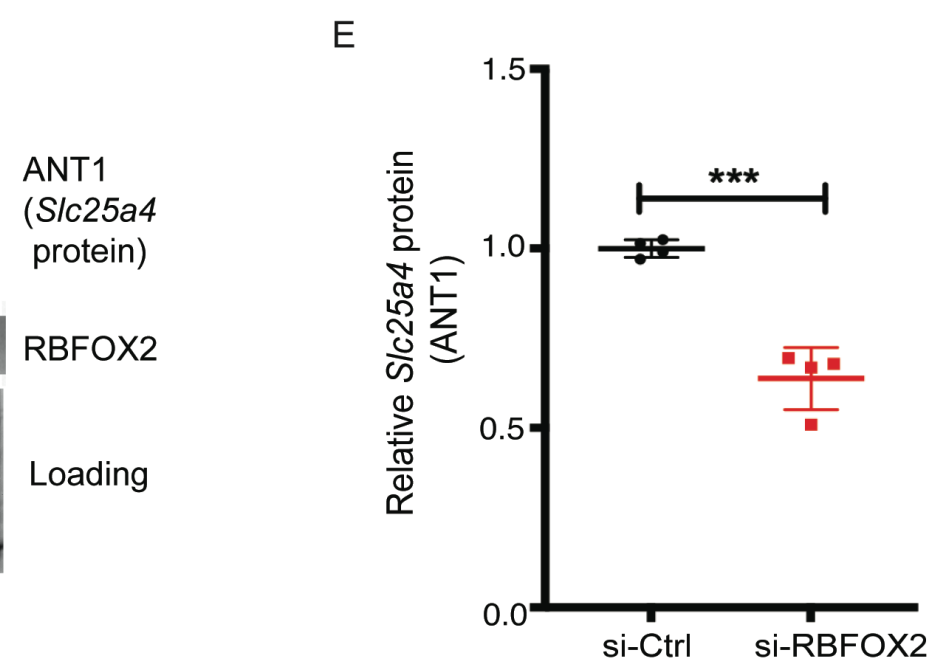




\section{Figure 5.}

(A) Cartoon representation of Luciferase-Slc25a4 polyA (pA) reporter constructs that harbor both pPAS and dPAS of S/c25a4 in the 3'UTR with or without mutated RBFOX2 binding motifs (underlined) located downstream of dPAS.

(B) RT-qPCR analysis of dPAS usage when normalized to total mRNA levels in HEK293 cells expressing WT or RBFOX2 binding site mutant pA Luciferase-SIc25a4 3'UTR constructs, n.6, $p<0.0001$ (three independent experiments).

(C) Relative firefly luciferase levels in HEK293 cells expressing WT or RBFOX2 binding site mutant pA Luciferase-S/c25a4 3'UTR constructs, $n>5, p<0.0001$ (three independent experiments). 


\section{Figure 5}

A

SIC25a4 3'UTR polyA reporters

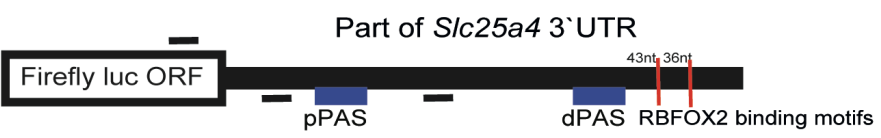

IPAS RBFOX2 binding motif

RBFOX2 binding motif

S/c25a4 3'UTR WT AGTACACTCCATCTCCCTGAACTCATTAACGACTAACAGATTGAATGCATCAATGCATGTTTTCTAGCAGGACACATTTGTCAGTGACTCTGGTGTGCATGCCCTGTACTGAACACGTTATAGGTTT SIC25a4 3'UTR MUTAGTACACTCCATCTCCCTGAACTCATTAACGACTAACAGATTGAATGCATCAATGACTGTTTTCTAGCAGGACACATTTGTCAGTGACTCTGGTGTGACTGCCCTGTACTGAACACGTTATAGGTTT

B

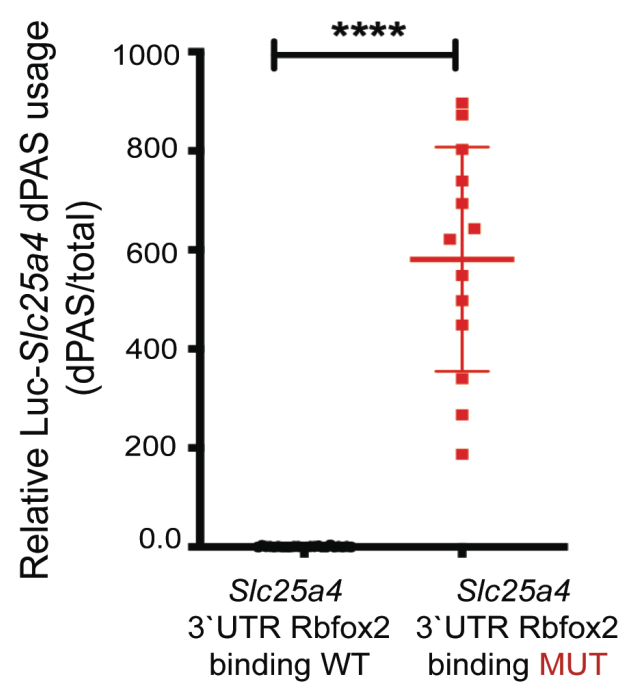

C

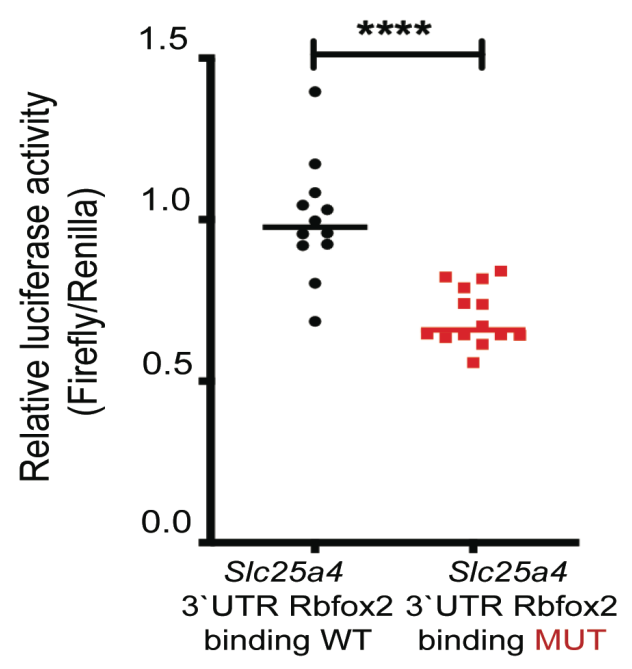


Figure 6. RBFOX2 depletion in H9c2 myoblasts affects mitochondrial health.

(A) Mitochondrial membrane potential $(\psi \mathrm{m})$ was determined by JC-1 staining of the $\mathrm{H} 9 \mathrm{c} 2$ cells transfected with scrambled or Rbfox 2 siRNA. Shown are the ratios of fluorescence intensity of J-aggregates (red fluorescence) to J-monomers (green fluorescence). Statistical significance was calculated using unpaired t-test to compare two different groups in five replicates of two independent experiments. Data represent means $\pm \mathrm{SD}$. For mitochondrial membrane potential in untreated control vs RBFOX2-KD H9c2 cells, ${ }^{* * * *} p<0.0001$; in control vs RBFOX2-KD H9c2 cells treated with Rotenone, ${ }^{* * *} p=0.0004$; in control vs RBFOX2-KD H9c2 cells treated with Antimycin $A,{ }^{* * *} p=$ 0.0009; in control vs RBFOX2-KD H9c2 cells treated with FCCP, ${ }^{* *} p=0.0017$; in control vs RBFOX2-KD H9c2 cells treated with $\mathrm{H}_{2} \mathrm{O}_{2},{ }^{* * * *} \mathrm{p}<0.0001$.

(B) Immunoflourescence of mitochondrial markers OPA1 and (C) MFN1 in RBFOX2 KD H9c2 cells imaged by confocal microscopy.

(D) Representative transmission electron microscopy images of mitochondria in control vs RBFOX2-KD H9c2 cells. Black arrows mark the mitochondria. Scale bar= 0.1 micron

(E) Surface area of mitochondria in control and RBFOX2 depleted myoblasts was calculated using Image J. ( $\mathrm{n}=234$ mitochondria for control, $\mathrm{n}=216$ mitochondria for RBFOX2 KD myoblasts), ${ }^{* * * *} p<0.0001$. 
Figure 6

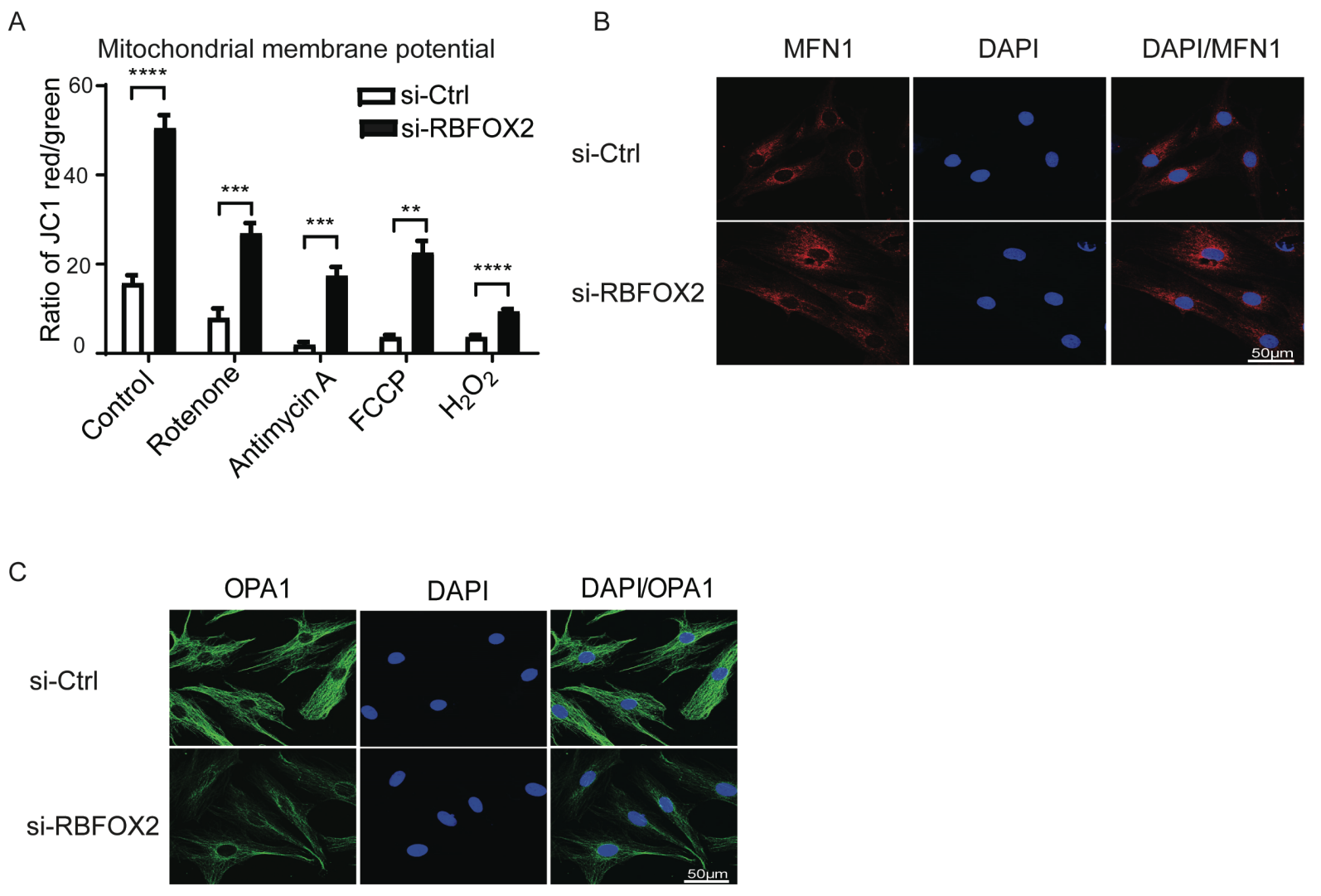

D

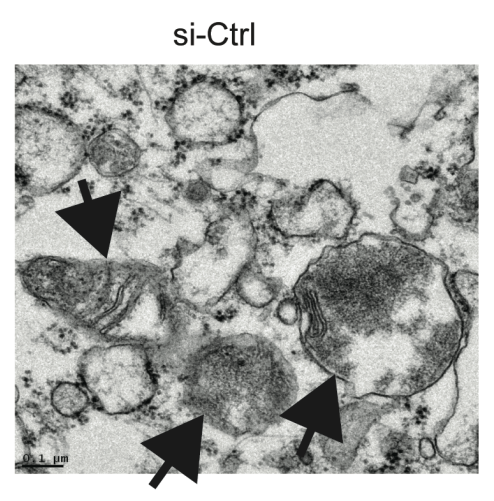

E

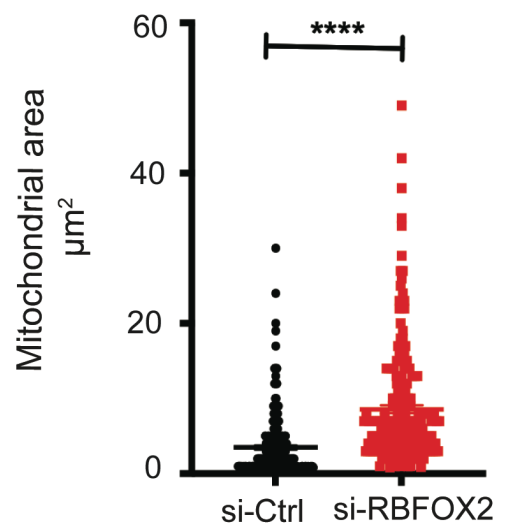




\section{SUPPLEMENTAL INFORMATION}

\section{Supplemental Figure 1.}

Average read lengths vs read quality of nanopore cDNA sequencing in $\mathrm{H} 9 \mathrm{c} 2$ myoblasts. 
bioRxiv preprint doi: https://doi.org/10.1101/2020.05.13.093013; this version posted March 25, 2021. The copyright holder for this preprint (which was not certified by peer review) is the author/funder. All rights reserved. No reuse allowed without permission.

Supplemental Figure 1

Read lengths vs Average read quality plot

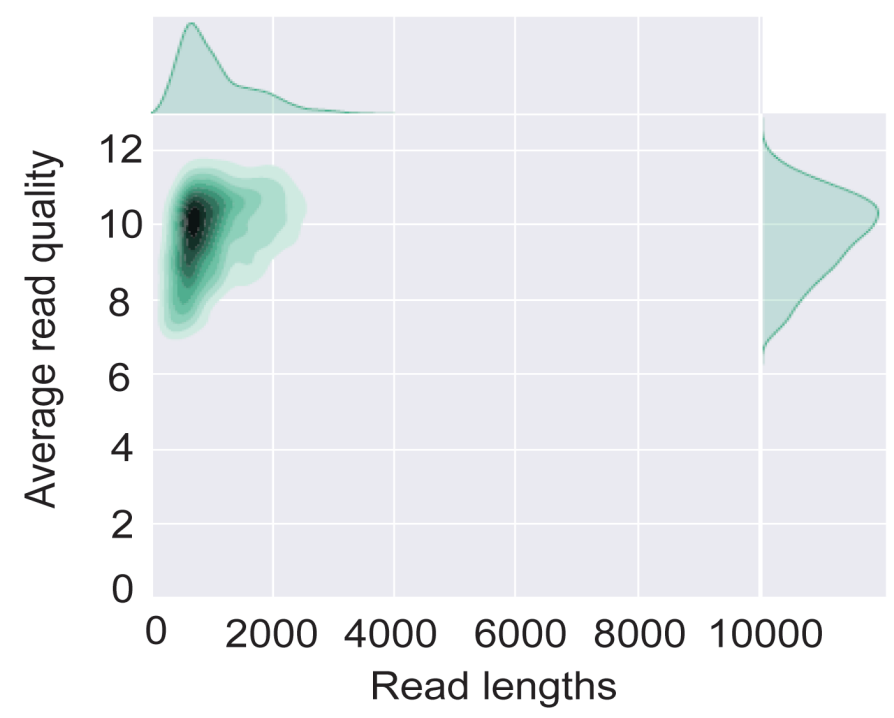


bioRxiv preprint doi: https://doi.org/10.1101/2020.05.13.093013; this version posted March 25, 2021. The copyright holder for this preprint (which was not certified by peer review) is the author/funder. All rights reserved. No reuse allowed without permission.

\section{Supplemental Figure 2.}

Full-length Tpm1 transcripts identified by nanopore cDNA sequencing in $\mathrm{H} 9 \mathrm{c} 2$ cells. 
Supplemental Figure 2

Tpm1 transcripts in control H9c2 cells

\begin{tabular}{|c|c|c|c|c|c|c|c|c|c|}
\hline 1 & $1 a$ & $2 b$ & 3 & 4 & 5 & $6 b$ & 7 & 8 & $9 d$ \\
\hline 2 & $1 \mathrm{a}$ & $2 b$ & 3 & 4 & 5 & $6 b$ & 7 & 8 & $9 a$ \\
\hline 3 & $1 a$ & $2 b$ & 3 & 4 & 5 & $6 b$ & 7 & 8 & \\
\hline 4 & $1 a$ & $2 a$ & 3 & 4 & 5 & $6 b$ & 7 & 8 & $9 d$ \\
\hline 5 & $1 a$ & $2 b$ & 3 & 4 & 5 & $6 b$ & 7 & 8 & $9 a$ \\
\hline 6 & & $2 b$ & 3 & 4 & 5 & $6 b$ & 7 & 8 & $9 d$ \\
\hline 7 & $1 a$ & $2 b$ & 3 & 4 & 5 & $6 b$ & 7 & 8 & $9 a$ \\
\hline 8 & $1 a$ & $2 b$ & 3 & $9 d$ & & & & & \\
\hline 9 & $1 a$ & $2 b$ & 3 & 4 & 5 & $6 a$ & 7 & 8 & $9 a$ \\
\hline 10 & $1 a$ & $2 b$ & 3 & 4 & 5 & 7 & 8 & $9 d$ & \\
\hline 11 & $1 \mathrm{a}$ & $2 b$ & 3 & 4 & 5 & $6 b$ & 7 & $9 a$ & \\
\hline 12 & $1 a$ & $2 b$ & 3 & 4 & 7 & 8 & $9 a$ & & \\
\hline 13 & $1 a$ & $2 a$ & 3 & 4 & 5 & $6 a$ & 7 & 8 & $9 d$ \\
\hline 14 & $1 a$ & $2 b$ & 3 & 4 & 5 & $6 b$ & 7 & & \\
\hline 15 & $1 \mathrm{a}$ & $2 b$ & 3 & 4 & 5 & $6 b$ & 7 & $9 a$ & $9 b$ \\
\hline 16 & $1 \mathrm{a}$ & $2 b$ & 3 & 4 & $6 b$ & 7 & 8 & $9 d$ & \\
\hline 17 & & $2 b$ & 3 & 4 & $6 b$ & 7 & 8 & $9 d$ & \\
\hline 18 & & $1 b$ & 3 & 4 & 5 & $6 a$ & 7 & 8 & $9 a$ \\
\hline 19 & & $2 b$ & 3 & 4 & 5 & $6 b$ & 7 & 8 & $9 a$ \\
\hline 20 & $1 \mathrm{a}$ & $2 b$ & 3 & 4 & 7 & 8 & $9 d$ & & \\
\hline 21 & $1 a$ & $2 b$ & 3 & 4 & $9 d$ & & & & \\
\hline 22 & $1 a$ & $2 b$ & 3 & 4 & 5 & $6 a$ & 7 & 8 & \\
\hline
\end{tabular}




\section{Supplemental Figure 3.}

Full-length Tpm1 transcripts identified by nanopore cDNA sequencing in RBFOX2 knocked down H9c2 cells.

Supplemental Figure 3

Tpm1 transcripts in RBFOX2 KD H9c2 cells

\begin{tabular}{|c|c|c|c|c|c|c|c|c|c|}
\hline $1^{\prime}$ & $1 a$ & $2 \mathrm{~b}$ & 3 & 4 & 5 & $6 a$ & 7 & 8 & $9 \mathrm{~d}$ \\
\hline $2^{\prime}$ & $1 a$ & $2 b$ & 3 & 4 & 5 & $6 b$ & 7 & 8 & $9 \mathrm{~d}$ \\
\hline $3^{\prime}$ & 1a & $2 b$ & 3 & 4 & 5 & $6 a$ & 7 & 8 & \\
\hline $4^{\prime}$ & 1a & $2 b$ & 3 & 4 & 5 & $6 a$ & 7 & $9 d$ & \\
\hline $5^{\prime}$ & 1a & $2 b$ & 3 & 4 & 5 & $6 b$ & 7 & 8 & \\
\hline $6^{\prime}$ & $1 \mathrm{a}$ & $2 \mathrm{~b}$ & 3 & 4 & 5 & $6 b$ & 7 & 8 & $9 a$ \\
\hline $7^{\prime}$ & 1a & $2 b$ & 3 & $9 \mathrm{~d}$ & & & & & \\
\hline $8^{\prime}$ & $1 \mathrm{a}$ & $2 \mathrm{~b}$ & 3 & 4 & $6 a$ & 7 & 8 & $9 d$ & \\
\hline $9^{\prime}$ & & $2 b$ & 3 & 4 & 5 & $6 a$ & 7 & 8 & $9 \mathrm{~d}$ \\
\hline
\end{tabular}

Reads

$\begin{array}{cc}\text { Experiment1 } & \text { Experiment2 } \\ 55 & 43 \\ 15 & 10 \\ 8 & 4 \\ 4 & 4 \\ 3 & 1 \\ 2 & 1 \\ 2 & 1 \\ 2 & 1 \\ 1 & 1\end{array}$


Supplemental Figure 4. Full length $T p m 2, T p m 3$, and Tpm4 transcripts do not display APA changes in RBFOX2 depleted H9c2 cells determined by nanopore sequencing. (A) Tpm2 transcript sequences mapped to the rat genome after nanopore sequencing in control and RBFOX2 depleted cells. (B) Tpm3 transcript sequences mapped to the rat genome after nanopore sequencing in control and RBFOX2 depleted cells. (C) Tpm4 transcript sequences mapped to the rat genome after nanopore sequencing in control and RBFOX2 KD cells. 
Supplemental Figure 4

A

\section{Rat Tpm2}

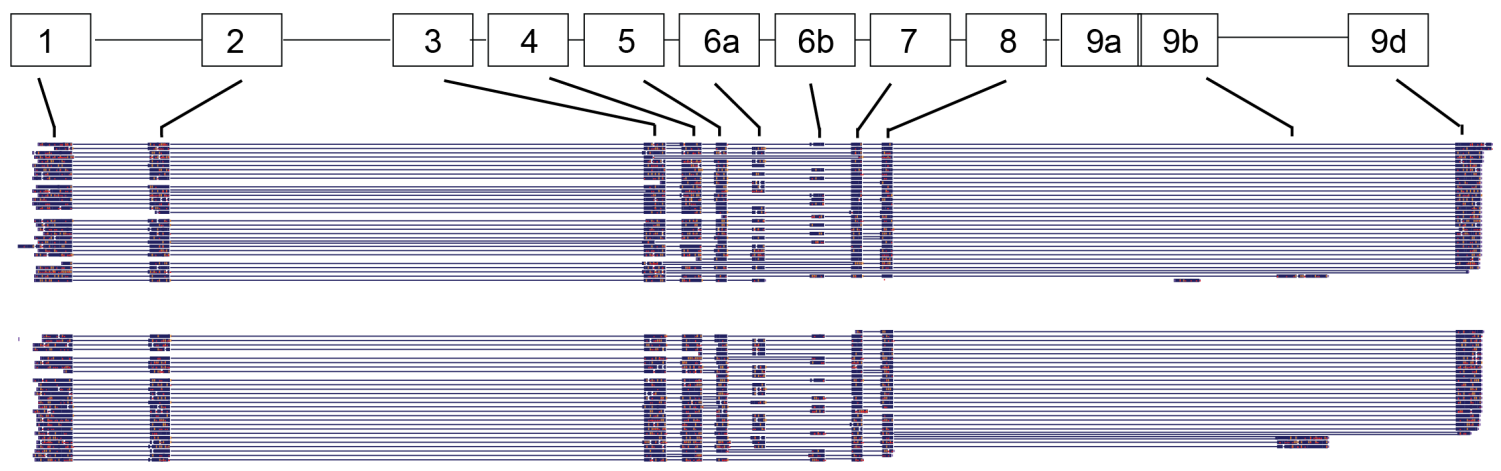

B

\section{Rat Tpm3}

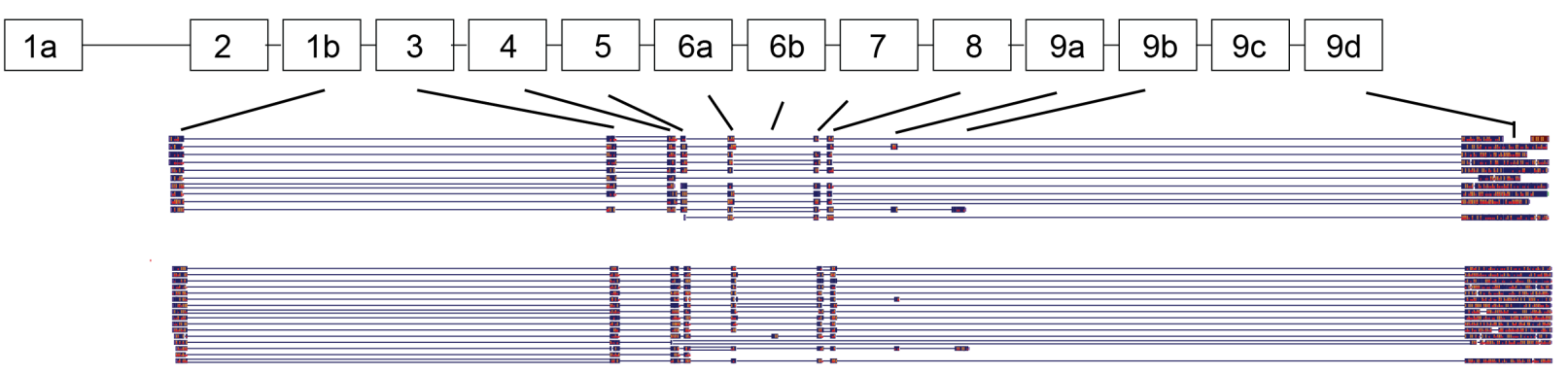

C

Rat Tpm4

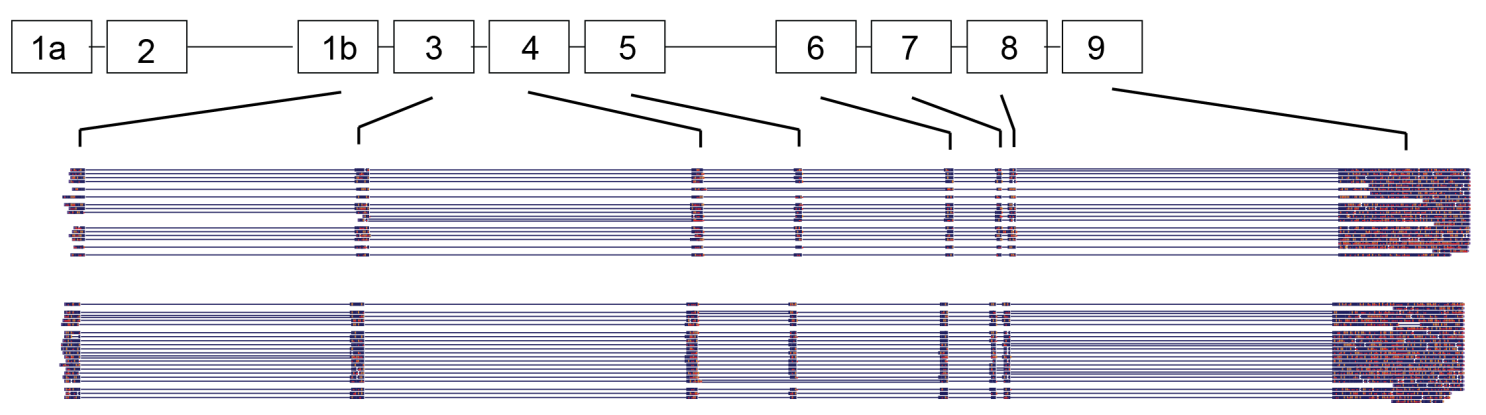


bioRxiv preprint doi: https://doi.org/10.1101/2020.05.13.093013; this version posted March 25, 2021. The copyright holder for this preprint (which was not certified by peer review) is the author/funder. All rights reserved. No reuse allowed without permission.

Supplemental Figure 5. SIc25a4 PAC-seq and nanopore sequencing data obtained from 3 different control and 3 different RBFOX2 KD H9c2 myoblasts. 


\section{Supplemental Figure 5}

PAC-Seq

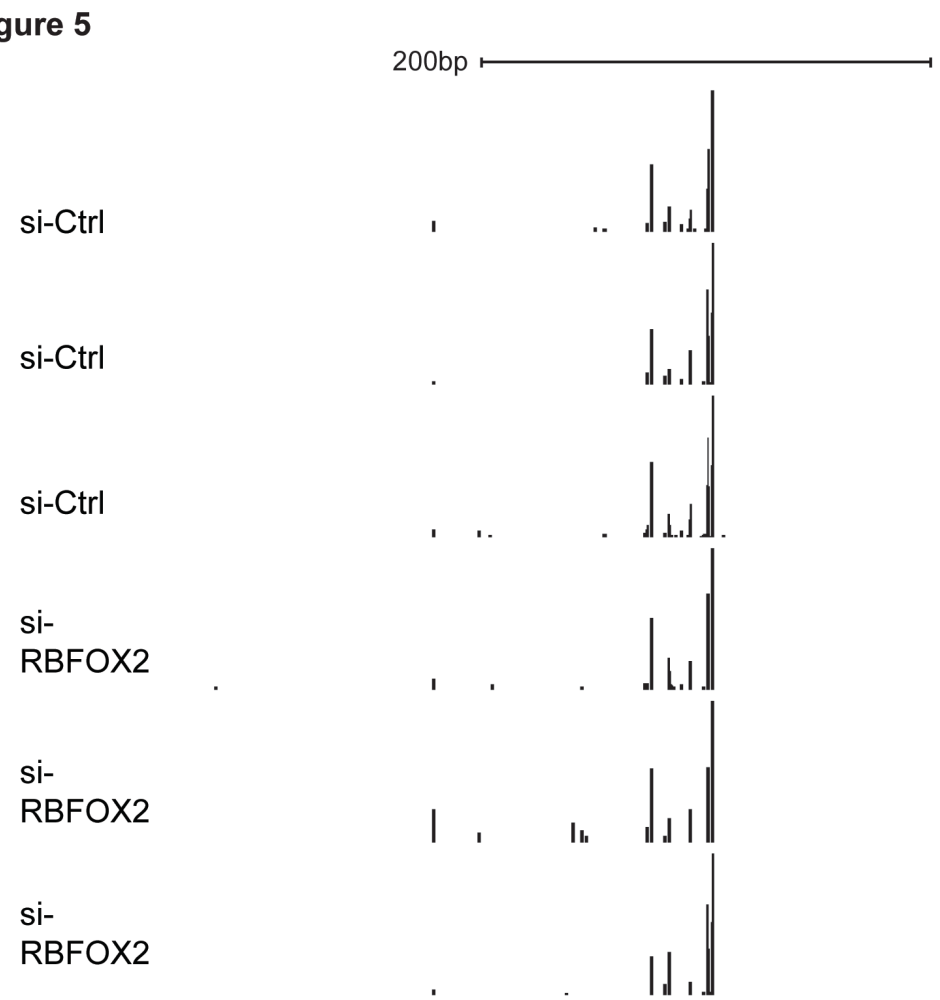

Nanopore

sequencing
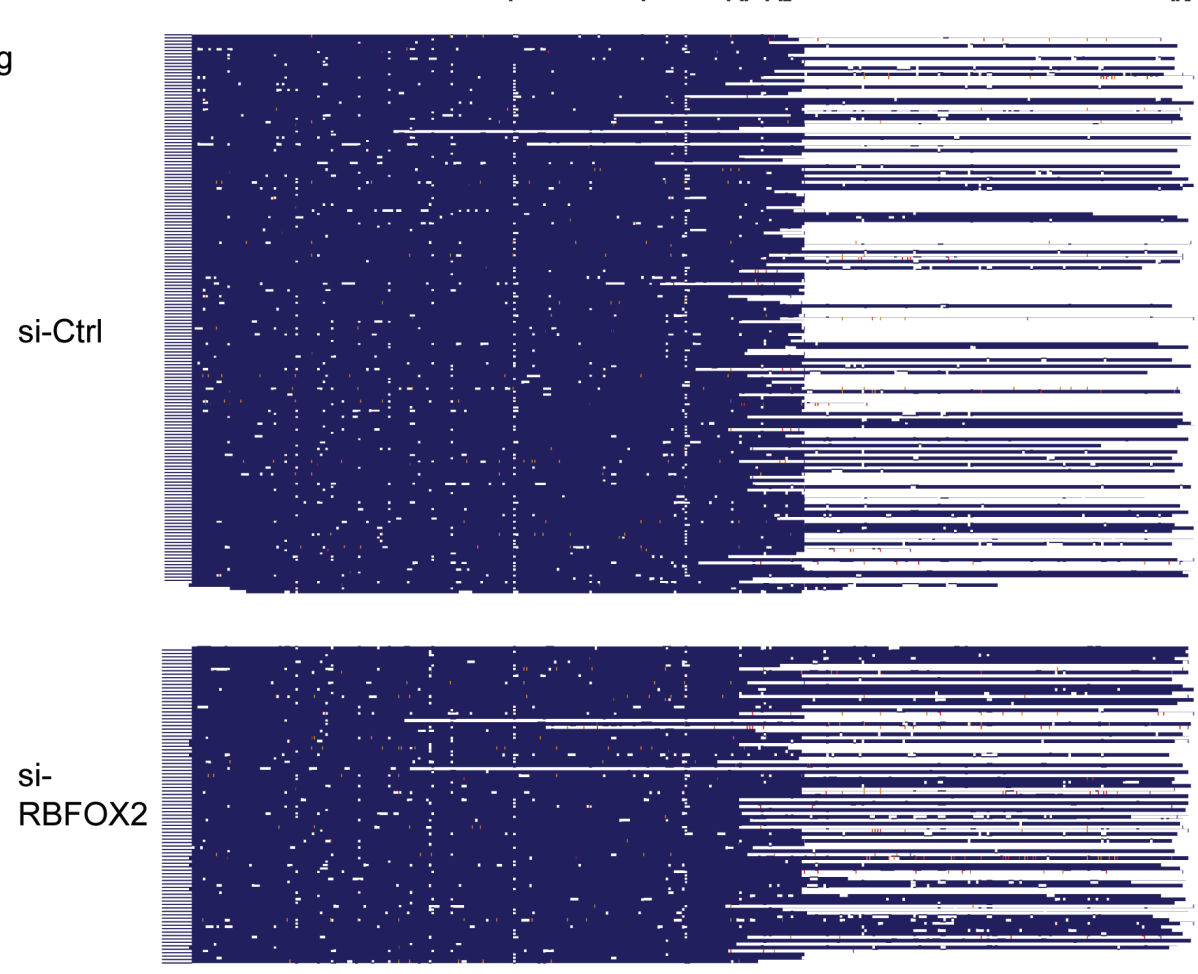


\section{Supplemental Tables}

Supplemental Table 1: Mapped reads and number of transcripts determined by nanopore sequencing after mapping to rn6 genome using Minimap2.

\begin{tabular}{|c|c|c|c|c|}
\hline & Raw Reads & Mapped Reads & Number of & Number of \\
& & & transcripts & transcripts \\
& & & $\mathrm{n}>5$ & 400 \\
\hline Ctrl_1 & 757587 & 737213 & 4316 & 413 \\
\hline Ctrl_2 & 520312 & 510766 & 3247 & 306 \\
\hline siRbfox2_1 & 370827 & 364183 & 3630 & 229 \\
\hline siRbfox2_2 & 317562 & 311204 & 3081 & 190 \\
\hline
\end{tabular}


Supplemental Table 2: Primer information

\begin{tabular}{|c|c|c|c|c|c|}
\hline $\begin{array}{l}\text { Primer } \\
\text { name }\end{array}$ & $\begin{array}{l}\text { Spe } \\
\text { cies }\end{array}$ & Gene ID & $\begin{array}{l}\text { Target } \\
\text { location }\end{array}$ & $\begin{array}{l}\text { Forward primer } \\
\left(5^{\prime} \text { to } 3^{\prime}\right)\end{array}$ & $\begin{array}{l}\text { Reverse primer (5‘ } \\
\left.\text { to } 3^{\prime}\right)\end{array}$ \\
\hline $\begin{array}{l}\text { Tpm1- } \\
\text { short }\end{array}$ & Rat & $\begin{array}{l}\text { ENSRNO } \\
\text { G0000001 } \\
8184\end{array}$ & $\begin{array}{l}\text { Exon7- } \\
\text { exon9b }\end{array}$ & $\begin{array}{l}\text { GAAGACAAATA } \\
\text { TGAAGAGGAGA } \\
\text { TCAAG }\end{array}$ & $\begin{array}{l}\text { AATAGCAAACAG } \\
\text { GAATAGCGTACA }\end{array}$ \\
\hline Tpm1-long & Rat & $\begin{array}{l}\text { ENSRNO } \\
\text { G0000001 } \\
8184 \\
\end{array}$ & $\begin{array}{l}\text { Exon7- } \\
\text { exon9d }\end{array}$ & $\begin{array}{l}\text { GAAGACAAATA } \\
\text { TGAAGAGGAGA } \\
\text { TCAAG }\end{array}$ & $\begin{array}{l}\text { CTGGTGCATACT } \\
\text { AAGGTTTTCTTCT } \\
\text { TT }\end{array}$ \\
\hline Hprt & Rat & $\begin{array}{l}\text { ENSRNO } \\
\text { T0000004 } \\
5153.3 \\
\end{array}$ & $\begin{array}{l}\text { Exon2- } \\
\text { exon3 }\end{array}$ & $\begin{array}{l}\text { CTCATGGACTG } \\
\text { ATTATGGACAG } \\
\text { GAC }\end{array}$ & $\begin{array}{l}\text { GCAGGTCAGCAA } \\
\text { AGAACTTATAGC } \\
\text { C }\end{array}$ \\
\hline $\begin{array}{l}\text { Slc625a4- } \\
\text { total }\end{array}$ & Rat & $\begin{array}{l}\text { ENSRNO } \\
\text { T0000001 } \\
4704.4\end{array}$ & $\begin{array}{l}\text { Exon4 - } \\
\text { 3`UTR }\end{array}$ & $\begin{array}{l}\text { CAACGTACTGA } \\
\text { GAGGCATGG }\end{array}$ & $\begin{array}{l}\text { CTGTGAACCTGT } \\
\text { GAACTTGAGC }\end{array}$ \\
\hline $\begin{array}{l}\text { Slc25a4- } \\
\text { long }\end{array}$ & Rat & $\begin{array}{l}\text { ENSRNO } \\
\text { T0000001 } \\
4704.4\end{array}$ & $\begin{array}{l}\text { Exon4 - } \\
\text { 3`UTR }\end{array}$ & $\begin{array}{l}\text { CAACGTACTGA } \\
\text { GAGGCATGG }\end{array}$ & $\begin{array}{l}\text { GTGGTTAAATGA } \\
\text { ATACTCAATG }\end{array}$ \\
\hline $\begin{array}{l}\text { Firefly } \\
\text { luciferase- } \\
\text { Slc25a4 } \\
\text { reporter- } \\
\text { long }\end{array}$ & & & $\begin{array}{l}\text { Firefly } \\
\text { luciferase- } \\
\text { Slc25a4 } \\
\text { 3UTR }\end{array}$ & $\begin{array}{l}\text { GTGGACGAGGT } \\
\text { GCCTAAAG }\end{array}$ & $\begin{array}{l}\text { CTT CTG TTT } \\
\text { GCT GTG GGA } \\
\text { ATC }\end{array}$ \\
\hline $\begin{array}{l}\text { Firefly } \\
\text { luciferase- } \\
\text { Slc25a4 } \\
\text { reporter- } \\
\text { total }\end{array}$ & & & $\begin{array}{l}\text { Firefly } \\
\text { luciferase- } \\
\text { Slc25a4 } \\
\text { 3.UTR }\end{array}$ & $\begin{array}{l}\text { GTGGACGAGGT } \\
\text { GCCTAAAG }\end{array}$ & $\begin{array}{l}\text { CAA TGG ATC } \\
\text { TGT GAA CCT } \\
\text { GTG }\end{array}$ \\
\hline $\begin{array}{l}\text { Renilla } \\
\text { Luciferase }\end{array}$ & & & & $\begin{array}{l}\text { TGG AGC CAT } \\
\text { TCA AGG AGA } \\
\text { AG }\end{array}$ & $\begin{array}{l}\text { CGA AGG TAG } \\
\text { GCG TTG TAG TT }\end{array}$ \\
\hline
\end{tabular}




\section{STAR METHODS}

\section{Cell culture}

H9c2 myoblast cells (ATCC CRL-1446) were cultured and maintained in Dulbecco's modified Eagle's medium (DMEM) (ATCC 30-2002), supplemented with 10\% fetal bovine serum (FBS, ATCC 30-2020) and 100 units/ml penicillin and streptomycin (Thermofisher Scientific 15140122). HEK293 cells were maintained in DMEM (Corning 10-013) supplemented with 10\% FBS (Corning 35-075), and 100 units/ml penicillin and streptomycin (Thermofisher Scientific 15140122). For siRNA-KD experiments, H9c2 cells were seeded at $10^{6}$ cells per $100 \mathrm{~mm}$ dish and transfected with scrambled siRNA (Thermofisher Scientific AM4611) or Rbfox2 siRNA (Thermofisher Scientific siRNA ID\# s96620) at 20nM using Lipofectamine RNAiMAX (Thermofisher Scientific 13778150). Cells were harvested 72 hours post-transfection for RNA or protein extraction. For rescue experiments, $3 \times 10^{6} \mathrm{H} 9 \mathrm{c} 2$ cells were transfected with eGFP (Sigma-Aldrich), human GFPRBFOX2 (transcript variant 3) (Addgene, plasmid \#63086) or empty vector (pcDNA 5) together with scrambled or Rbfox 2 specific siRNAs by Neon Nucleofection System (Thermofisher Scientific) and harvested 48 hours post-transfection as we have described (Verma et al., 2013).

\section{RNA}

RNA was extracted from cells using TRIzol (Thermofisher Scientific 15596-018) by following the manufacturer's protocol with the exception that RNA was precipitated overnight at $-70^{\circ} \mathrm{C}$. For PAC-seq, the amount and quality of RNA were analyzed by using an Agilent bioanalyzer at the University of Texas Medical Branch Next-Generation 
Sequencing Core Facility. For nanopore sequencing, poly $(A)+$ RNA was enriched by using magnetic mRNA isolation kit (New England biolabs S1550S).

\section{Poly(A)-ClickSeq (PAC-seq)}

The PAC-seq protocol is previously described by Routh et al (Routh et al., 2017). Three samples per treatment/biological condition were used for the PAC-seq analysis. Briefly, $2 \mu \mathrm{g}$ of total RNA was used to synthesize cDNA through standard reverse transcription initiated by Illumina $4 \mathrm{~N} \_21 \mathrm{~T}$ primer p7 and terminated by incorporation of azidonucleotides (AzVTPs). Subsequently, the cDNA was cleaned by addition of RNase $\mathrm{H}$ (NEB) and purified through DNA Clean and Concentrator Kit (Zymo) before click-reaction, in which the 5' hexynyl- "click-adapter" p5 was click-ligated with azido-terminated cDNA in the presence of copper-TBTA (Lumiprobe) and Vitamin C. After DNA purification, the clicked cDNA was mixed with indexing primer, universal primer complementary to $p 7$ and One Taq Standard Buffer Master Mix for PCR amplification. PAC-seq libraries were submitted for $1 \times 150$ single end sequencing yielding between 14 and 25 million reads per sample. The output data was processed and mapped to the rattus norvegicus genome (rn6) and poly(A)-clusters (PACs) were generated using the DPAC pipeline (Routh, 2019b), designed for automated analysis and annotation of poly(A)-targeted RNAseq libraries such as PAC-seq.

DPAC (Routh, 2019b) uses DESeq2 (Love et al., 2014) to measure changes in PAC usage between control and RBFOX2 KD cells. Data processing, read mapping and poly(A)-site detection were performed using the default parameters of the DifferentialPoly(A)-Clustering (DPAC) pipeline (Routh, 2019a). PASs are defined as the exact 
nucleotide of the 3'UTR to poly $(A)$ tract junction found within individual sequencing reads, while poly(A)-clusters (PACs) are defined as clusters of PASs when they are found within $<10$ nts of one another (default parameter in DPAC pipeline). The differential PAC usage was reported if an individual PAC comprises at least $5 \%$ of a gene's PACs, has a fold change of $>1.5$, and results with an Independent Hypothesis Weighted (Ignatiadis et al., 2016) multiple testing p-adjusted value of $<0.1$.

\section{Nanopore sequencing with MinION}

For nanopore sequencing, two sets of control and RBFOX2 KD H9c2 cells were used. Total cellular RNA was first poly(A) enriched and then amplified using oligo-dT primers and template switching oligos using Oxford Nanopore Technologies (ONT) cDNA-PCR sequencing kit (PCS108) as prescribed by the manufacturer. Samples were multiplexed using ONT barcodes. Pooled samples were sequenced on R9.4 flow cells. Reads were demultiplexed and base-called using Albacore followed by mapping to the rat genome (rn6) using the splice function of minimap2 (Li, 2016). Transcript isoforms were identified using FLAIR (Tang et al., 2018).

\section{JC-1 Mitochondrial membrane potential $(\Psi \mathrm{m})$ assay}

H9c2 cells were seeded on to 96-well black flat bottom plates (Costar). After 24 hours, cells were incubated in DMEM media without FBS and phenol red at $37^{\circ} \mathrm{C} / 5 \% \mathrm{CO}_{2}$ with $5 \mu \mathrm{M}$ rotenone (inhibits complex I) or $30 \mu \mathrm{M}$ antimycin A (inhibits complex III) for 24 hours. In some experiments, cells were also incubated with $250 \mu \mathrm{M}$ trifluoromethoxy 
carbonylcyanide phenylhydrazone (FCCP, uncoupler of membrane permeability and oxidative phosphorylation) or $400 \mu \mathrm{M} \mathrm{H}_{2} \mathrm{O}_{2}$ that was added during last 4 hours of incubation. Plates were washed with PBS and then $100 \mu \mathrm{l}$ of $10 \mu \mathrm{g} / \mathrm{ml} \mathrm{JC}-1\left(5,5^{\prime}, 6,6^{\prime}-\right.$ tetrachloro-1,1',3,3'-tetraethylbenzimidazolocarbocyanine iodide, Invitrogen, T3168) added. Cells were incubated at $37^{\circ} \mathrm{C}$ for 15 minutes in dark, washed twice with PBS, and $100 \mu \mathrm{l}$ PBS was added to each well. Plates were read in SpectraMax M2 (Molecular Devices) to measure red $\mathrm{J}$-aggregates fluorescence (a sensitive marker of $\Psi \mathrm{m}$ ) at an excitation of $535 \mathrm{~nm}$ and emission of $595 \mathrm{~nm}$ and green J-monomers fluorescence (indicator of disruption of $\Psi_{\mathrm{m}}$ ) at an excitation of $485 \mathrm{~nm}$ and emission of $535 \mathrm{~nm}$. Ratio of red/green fluorescence was calculated and plotted on the graph.

\section{RT-qPCR}

Briefly, a master mix was set up by mixing $5 \mu$ of cDNA, $3 \mu$ of $\mathrm{H}_{2} \mathrm{O}, 2 \mu \mathrm{l}$ of PCR gene specific primer (10X conc) and $10 \mu$ l of master mix (Roche 04707516001) in $20 \mu l$ reaction. The qRT-PCR was conducted using LightCycler 480 Instrument (Roche) using the following conditions: $95^{\circ} \mathrm{C} 10 \mathrm{~s} ; 62^{\circ} \mathrm{C} 15 \mathrm{~s} ; 72{ }^{\circ} \mathrm{C} 10 \mathrm{~s}$ for 40 cycles. Melting curve was obtained to ensure single product. $\Delta \mathrm{Ct}$ method was adopted for quantification. qPCR quantifications were as described previously (Belanger et al., 2018; Nutter et al., 2016b; Verma et al., 2016b). For luciferase-Slc25a4 reporter qPCRs, forward primer was designed on the firefly luciferase ORF, and reverse primers were designed to bind SIc25a4 3`UTR in different locations (Supp. Table 2). Firefly-SIc25a4 heterologous mRNA generated via dPAS usage in comparison to total Firefly-SIc25a4 heterologous mRNA was normalized to renilla luciferase mRNA levels. Semi-quantitative RT-PCR was used 
for determining Tpm1 (short and long) transcripts due to the generation of 2 distinct DNA bands representing short and long isoforms from the same PCR reaction. $2 \mu \mathrm{g}$ of total

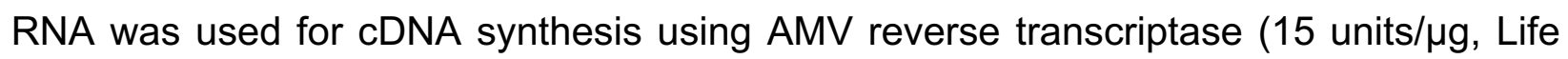
Biosciences). PCR was performed using $5 \mu$ l of cDNA, $25 \mu \mathrm{M}$ dNTPs, 100ng of each gene specific forward and reverse primer and $0.2 \mu$ of Biolase Taq polymerase (Bioline) in a $20 \mu \mathrm{l}$ reaction. All primer sequences are provided in Supp. Table 2.

\section{Western blot}

Cells were lysed in lysis buffer (10mM HEPES-KOH, pH7.5, $0.32 \mathrm{M}$ Sucrose, $5 \mu \mathrm{M}$ MG132, 5mM EDTA, 1.0\% SDS, and proteinase inhibitor cocktail from Roche) and sonicated. Protein concentration was determined by using the Bicinchoninic acid assay (BCA, Sigma BCA1-1KT). $30 \mu \mathrm{g}$ of protein was separated on $10 \%$ SDS-PAGE and transferred to a PVDF membrane (Immobilon-P, Millipore). The membrane was blocked with $5 \%$ dry fat-free milk solution in PBS containing $0.1 \%$ Tween (PBST) at RT for 1 hour and then incubated with indicated primary antibodies overnight at $4^{\circ} \mathrm{C}$. The membrane was washed with PBST for three times and incubated with HRP-conjugated secondary antibody for 1 hour at RT followed by three washes using PBST. Immobilon Western chemiluminescent (Millipore WBKLS0500) kit was used to detect HRP activity of the secondary antibody. The membrane was then imaged using ChemiDoc Touch imaging system (Bio-Rad). Image J software was used for band intensity quantification. Primary antibodies used for this study are as follows: RBFOX2 (1:1000, Abcam, ab57154), TPM1 (1:1000, Cell Signaling, D12H4), a-tubulin (1:20000, Sigma-Aldrich, T6074) and ANT1:Slc25a4 (1/750, Abcam, ab102032). 


\section{Immunofluorescence of mitochondrial proteins}

H9C2 cells were seeded on to the Nunc lab-tek chamber slides for overnight and then fixed with 4\% paraformaldehyde in PBS for 10 minutes followed by permeabilization and blocking in $0.1 \%$ triton $\mathrm{X} 100$ in $10 \%$ goat serum for 2 hours. Cells were then incubated overnight with rabbit anti-OPA1 (Abcam ab157457) and mouse anti-MFN1 (Abcam ab57602) monoclonal antibodies, diluted in $10 \%$ goat serum. Then, cells were washed with PBS, incubated with Alexa Flour 594-conjugated goat anti-mouse or Alexa Flour 488conjugated goat anti-rabbit secondary antibody, and slides were mounted using VECTASHIELD Antifade Mounting Medium with DAPI (Vector Laboratories, Burlingame, CA). Slides were imaged using Zeiss LSM 880 with Airyscan confocal microscope with 63X 1.4 oil immersion objective and images were processed using Image $\mathrm{J}$ software.

\section{Transmission Electron Microscopy}

To obtain ultrastructural analysis of cells in ultrathin sections, cells were fixed for at least 1 hour in a mixture of $2.5 \%$ formaldehyde prepared from paraformaldehyde powder, and $0.1 \%$ glutaraldehyde in $0.05 \mathrm{M}$ cacodylate buffer $\mathrm{pH} 7.3$ to which $0.01 \%$ picric acid and $0.03 \% \mathrm{CaCl}_{2}$ were added. Cell monolayers were washed in $0.1 \mathrm{M}$ cacodylate buffer, were scraped off and processed further as a pellet. The pellets were post-fixed in $1 \% \mathrm{OsO}_{4}$ in $0.1 \mathrm{M}$ cacodylate buffer $\mathrm{pH} 7.3$ for 1 hour, washed with distilled water and en bloc stained with $2 \%$ aqueous uranyl acetate for $20 \mathrm{~min}$ at $60^{\circ} \mathrm{C}$. The cell pellets were dehydrated in ethanol, processed through propylene oxide and embedded in Poly/Bed 812 (Polysciences, Warrington, PA). Ultrathin sections were cut on Leica EM UC7 
ultramicrotome (Leica Microsystems, Buffalo Grove, IL), stained with lead citrate and examined in a JEM-1400 (JEOL USA, Peabody, MA) transmission electron microscope at $80 \mathrm{kV}$. Digital images were acquired with a bottom-mounted CCD camera Orius SC200 1 (Gatan, Pleasanton, CA).

\section{Luciferase activity of SIc25a4 3'UTR constructs}

The 3'end of rat S/c25a4 3'UTR sequence, which contains multiple poly(A) sites (pPAS and pPAS) and RBFOX2 binding sites downstream of dPAS were cloned into pmirGLO Dual-luciferase vector (Promega, USA) downstream of firefly luciferase open reading frame (ORF). SV40 polyA site was removed in this plasmid to allow only the usage of polyA sites provided within the SIc25a4 3'UTR. Two RBFOX2 binding motifs near the distal poly(A) sites in the 3'UTR of S/c25a4 gene were abolished by mutating "CA" to "AC" (S/c25a4 3'UTR RBFOX2 binding WT or S/c25a4 3'UTR RBFOX2 binding MUT). 1X106 HEK293 cells seeded in 6 well plates $18 \mathrm{~h}$ before transfection, and transfected with 750 ng DNA (Luciferase SIc25a4 3'UTR polyA reporter constructs) using XtremeGene 9 (Sigma Aldrich, USA). Cells were lysed 24 hours after transfection and determined by the Dual luciferase assay kit (Promega, USA) and recorded using BioTek Cytation 5 plate reader. Firefly luciferase was normalized to renilla luciferase activity and compared between WT and mutant luciferase SIc25a4 3'UTR polyA reporter constructs. 